\title{
Políticos y periodistas: un bucle endogámico en los medios sociales: dominios compartidos en Twitter por diputados y periodistas españoles
}

\author{
Politics and journalism: a self-referential bubble in \\ social media: Twitter domains shared by \\ representatives and journalists in Spain
}

\author{
Pere FRANCH ${ }^{1}$ (D) 0000-0002-4180-1168 \\ Josep-Lluís MICÓ1 (iD) 0000-0003-1191-226X
}

\begin{abstract}
Resumen
Se presenta el resultado de una investigación sobre cómo se usan las redes sociales en comunicación política; en concreto, acerca de cómo utilizan Twitter los políticos y los periodistas en España. Mediante el machine learning y con una herramienta creada ad hoc para llevar a cabo un seguimiento del rastro digital (Digital Trace Data) se han observado y analizado los hiperenlaces que han compartido los diputados españoles de la XII legislatura y los principales periodistas del país, desde junio de 2018 hasta mayo de 2019. Los resultados revelan una relación endogámica entre periodistas y políticos. Así, la interacción tanto dentro de cada grupo como entre los dos se desarrolla alrededor de los principales medios españoles, por lo que ambos colectivos comparten información dentro de un bucle autorreferencial que provoca un efecto echo chamber y anula las posibilidades de expansión del conocimiento que proporcionan las redes sociales y el acceso al hipertexto.
\end{abstract}

Palabras clave: Comunicación política. Información sobre comunicación. Medios sociales. Periodismo. Política.

\begin{abstract}
This paper presents a study on how social media is used in political communication, specifically on how Twitter is used by politicians and journalists in Spain. Through a tool created ad hoc to monitor digital trace data, the authors observe - thanks to machine learning techniques - the hyperlinks that were shared both by the Spanish representatives in the XII National Congress and the main Spanish journalists from June 2018 to May 2019. The results reveal an endogenous relationship between journalists and politicians. The interaction between these two groups is produced in and around the main Spanish media, resulting in an inner flux of information shared by both groups within a self-referential bubble, thus producing an echo chamber effect and missing the enormous possibilities of knowledge expansion provided by social media through the accession to hypertext.
\end{abstract}

Keywords: Political communication. Communication information. Social media. Journalism. Politics.

1 Universidad Ramon Llull, Facultad de Comunicación y Relaciones Internacionales Blanquerna, Departamento de Periodismo. Plaça Joan Coromines, s/n., 08001, Barcelona, España. Dirección de correspondencia/Correspondence to: P. FRANCH. E-mail: <perefp@blanquerna.url.edu>.

Apoyo: Ministerio de Economía, Industria y Competitividad de España. Proyecto "Influencers en la comunicación política en España: Análisis de las relaciones entre líderes de opinión 2.0, medios de comunicación, partidos, instituciones y audiencias en el entorno digital" (CSO2017-88620-P).

Recibido el 19 del mayo del 2020, re-presentada el 19 del octubre del 2020 y aprobado el 3 del diciembre del 2020.

Como citar este artículo/How to cite this article

Franch, P.; Micó, J.-L. Políticos y periodistas: un bucle endogámico en los medios sociales: dominios compartidos en Twitter por diputados y periodistas españoles. Transinformação, v. 33, e200025, 2021. https://doi.org/10.1590/2318-0889202133e200025 


\section{Introducción}

Las redes sociales han devenido una de las principales herramientas para la comunicación política. El presidente norteamericano, Donald Trump, es el mejor ejemplo de político que recurre a Twitter para conectar directamente con el público y sortear así el carácter de mediador de los medios de comunicación, a los que denosta como enemigos del pueblo. Las redes son un factor determinante en la difusión (viralización) de información y pueden ser un campo ideal de ampliación del conocimiento. Twitter, en concreto, que es la red observada en el presente estudio, permite no solo mandar mensajes de texto de 280 caracteres, con fotografías, emojis, menciones y hashtags, sino que también permite compartir hiperenlaces a infinidad de fuentes.

Nos ha interesado observar qué uso hacen de Twitter los políticos y los periodistas en España. Para el presente estudio hemos observado en concreto qué dominios de Internet han sido los más compartidos a través de Twitter por los diputados de España elegidos para la XII legislatura y por periodistas destacados durante un periodo determinado: el que va desde el nombramiento del socialista Pedro Sánchez como presidente del Gobierno, el día 1 de junio de 2018, tras ganar la moción de censura presentada contra el gobierno conservador de Mariano Rajoy, hasta el 21 de mayo de 2019, día en que finaliza la XII legislatura porque se constituye el Congreso surgido de las urnas en las elecciones anticipadas del 28 de abril de aquel año.

El estudio consiste en realizar una taxonomía de los dominios compartidos en Twitter por más perfiles (diputados y periodistas) y los compartidos más veces, con una comparativa entre los dos grupos, el de los 331 miembros del Congreso de los diputados que tienen cuenta en la red social y el de los 158 periodistas seleccionados. Se observan dos variables de cada colectivo: el número de miembros de cada grupo que comparten los hiperenlaces y las veces que los comparten. Por tanto, más que partir de una hipótesis, la principal pregunta de investigación que nos planteamos vendría a formularse de la siguiente manera: ¿Qué dominios comparten diputados y periodistas españoles vía Twitter?, ¿con qué frecuencia o asiduidad? ¿qué coincidencias hay entre los dos grupos? y ¿qué aspectos aparecen en común y cuáles son claramente distintivos de los dos grupos? Otras posibles preguntas de investigación son: ¿Los diputados comparten mayoritariamente los dominios web de los medios o de los partidos? ¿Los dominios de medios más compartidos por los diputados coinciden con los de más audiencia entre la población española? ¿Hacen los periodistas un uso endogámico, autorreferencial, de las redes sociales al compartir los enlaces de los medios que ellos mismos dirigen o en los que participan? ¿Qué uso hacen de las otras principales redes sociales como Facebook, Instagram o Youtube? ¿Hay alguna distinción entre el uso de los dominios de los pure players o nativos digitales (por ejemplo, eldiario.es, publico.es, etc.) y los medios tradicionales o legacy media (El País, El Mundo, ABC, etc.)?

La importancia de las redes sociales en el ámbito de la comunicación es incontestable: a 30 de junio de 2020 tienen, según datos de We Are Social y Hootsuite, citados por EPdata (2020), 3.805 millones de usuarios en el mundo, 29 millones en España, donde el 53\% de usuarios de Internet afirma tener cuenta en Twitter. En España es la quinta red social más usada, detrás de Youtube, Whatsapp, Facebook e Instagram. En el periodo estudiado, Twitter tenía alrededor de 5 millones de usuarios: en 2018 fue la cuarta red con más usuarios (4,9 millones) pero en 2019 fue superada por Instagram y pasó al quinto lugar, a pesar de crecer hasta los 5,4 millones (Asociación para la Investigación de Medios de Comunicación, 2019).

\section{Estado del arte}

La aparición de la Web 2.0 rompió el monopolio de los actores políticos y los medios de comunicación en el proceso de producir y distribuir contenido (Chadwick, 2013). Las redes sociales han reescrito la fórmula de quién produce información política, quién la consume y cómo se distribuye (Gainous; Wagner, 2014), lo que ha dado lugar a la aparición de nuevas posibilidades de comunicación y acción política por parte de los tres actores clave en la comunicación política: políticos, periodistas y ciudadanos. 


\section{El uso de las redes por políticos y periodistas}

En la segunda década del siglo XXI, Twitter ha pasado a ser un espacio que las figuras políticas utilizan cada vez más para difundir sus mensajes e interactuar (o no) con otros agentes sociales. Desde la exitosa campaña del demócrata Barack Obama en las presidenciales estadounidenses de 2008, el uso de esta plataforma por parte de los actores políticos no ha dejado de aumentar, especialmente en las democracias occidentales (Gainous; Wagner, 2014).

La mayoría de las investigaciones sobre Twitter y política se centran en cuatro aspectos: Primero, la presencia de los actores políticos en esta plataforma. Los resultados sugieren que las campañas electorales estimulan el crecimiento en el número de políticos que se unen a Twitter (Graham et al., 2016; Campos-Domínguez, 2017) y su uso para fomentar su capacidad de liderazgo (López-García, 2016). Los aspectos que influyen en la decisión de utilizar esta red son diversos: los recursos económicos disponibles, la competitividad en el proceso electoral, la ideología del partido, el peso del partido en el contexto social y político y su posición en el gobierno o la oposición (Golbeck et al., 2010; Ausserhofer; Maireder, 2013).

El segundo aspecto se refiere a los hábitos de uso de los políticos en Twitter. Algunos estudios indican que lo utilizan principalmente como vehículo para la autopromoción, para diseminar información, lanzar campañas negativas y para la movilización partidista, sin hacer, por tanto, un uso interactivo de la red social (Golbeck et al., 2010; Enli; Skogerbø, 2013). A partir de la mitad de la década de 2010, los políticos populistas encuentran en Twitter un terreno donde prodigar su discurso (Van-Kessel; Castelein, 2016; Ernst et al., 2017). En este campo, y centrado en España, debe mencionarse el trabajo de Casero-Ripollés et al. (2017) sobre el populismo del espectro político de izquierdas. En su ampliación a Europa del estudio del fenómeno populista, Alonso-Muñoz y Casero-Ripollés (2018) descubren que hay muy poca diversidad temática y una relación entre el número de mensajes publicados y el interés de los usuarios que es inversamente proporcional.

En tercer lugar, sobre el contenido, alguna investigación indica que los políticos tuitean principalmente sobre asuntos personales (Jackson; Lilleker, 2011); esta estrategia tiene como objetivo reducir la brecha que separa a las élites políticas de los ciudadanos (Parmelee; Bichard, 2012). Otros estudios, sin embargo, muestran que los principales destinatarios de los mensajes de los políticos son periodistas y otros políticos, por lo que Twitter deviene un espacio de intercambio autorreferencial, una echo chamber para la élite política (Bruns; Highfield, 2013). Al observar a la vez a políticos y periodistas, Enli y Simonsen (2018) concluyen que a pesar de que ambos colectivos utilizan las redes sociales de manera distinta, coinciden sin embargo en el uso de hashtags y siguen la lógica de buscar contactar con públicos de fuera de sus seguidores y expandir así su influencia.

El cuarto aspecto está relacionado con la interacción entre Twitter y los principales medios de comunicación. La literatura sobre este aspecto indica que la agenda de los medios se ha transferido a las redes sociales cuando se trata de grandes eventos como debates electorales o convenciones políticas (Graham et al., 2016). No obstante, otros estudios indican que, en algunas ocasiones, son las redes sociales las que influyen en la composición de la agenda de los medios de comunicación tradicionales (Russell-Neuman et al., 2014).

Los periodistas que cubren temas políticos utilizan Twitter sobre todo para promocionar su medio y comentar con su público sus artículos y los de sus colegas, reforzando un uso endogámico de la red (Molyneux; Mourao, 2019). Otros estudios tratan temas más tangenciales o sectoriales; Bentivegna y Marchetti (2018) analizan cómo los periodistas italianos han adaptado a Twitter su actividad en relación con la transparencia, la función de gatekeeping y el compromiso con su público. Usher et al. (2018) comprueban en su estudio sobre los periodistas que cubren el Congreso de los Estados Unidos que en Twitter se mantiene el sesgo de género respecto a las mujeres. García-Perdomo (2017) analiza a los cien periodistas colombianos más influyentes en Twitter y concluye que a través de la red se implican profundamente en política al opinar abiertamente sobre la acción del expresidente Álvaro Uribe. 


\section{El uso de Twitter en los procesos electorales}

Los procesos electorales aumentan el uso de Twitter por parte de los políticos. De hecho, la mayor parte de los artículos publicados sobre esta cuestión se centran en periodos de campaña electoral (Golbeck et al., 2010; Vergeer; Hermans, 2013; Vergeer et al., 2013, Gainous; Wagner, 2014; Karlsen; Enjolras, 2016; Persily, 2017).

En su amplio repaso de la literatura acerca del uso de Twitter en los procesos electorales, Jungherr (2016) saca algunas conclusiones generales de 127 estudios académicos relevantes centrados en 26 países. En cuanto al uso por parte de partidos y candidatos, su investigación concluye que estos suelen usar Twitter principalmente para publicar información sobre sus actividades de campaña con links a sus propios sitios web (Graham et al., 2016). Si bien varios estudios observaron que algunos candidatos usan Twitter para publicar mensajes con contenido personal, la interacción con sus seguidores es muy poco habitual, por lo que los candidatos adoptan lo que podría definirse como un estilo puramente transmisor del uso de Twitter, reforzando así una comunicación unidireccional de líder a público (Jackson; Lilleker, 2011; Aharony, 2012; Adams; McCorkindale, 2013; Enli; Skogerbø, 2013). Por ello, Jungherr concluye que "hay poca evidencia de que Twitter sea un dispositivo que facilite el diálogo entre candidatos y ciudadanos normales" en las campañas electorales (Jungherr, 2016, p.76).

En cuanto a España, las investigaciones realizadas constatan que ni en periodos electorales aumenta la interacción de los políticos con los periodistas y los medios (López-Meri; Casero-Ripollés, 2016; Pérez-Dasilva et al., 2018). Al estudiar la campaña de las elecciones españolas de 2016, López-Meri et al. (2017) concluyen que los políticos usan Twitter para difundir sus propuestas políticas, pero sin recurrir a la personalización.

\section{Uso de las redes sociales por los ciudadanos y su efecto en los medios}

Es un hecho incontestable que cada vez más los ciudadanos dejan de lado los medios de comunicación y en su lugar acuden a las redes sociales para informarse: en las elecciones presidenciales de Estados Unidos de 2016, el 44\% de los norteamericanos recurrieron a las redes sociales para obtener información (Pew Research Center, 2016). Un estudio sobre las fuentes de información de los votantes estadounidenses muestra que la tercera fuente en las elecciones de 2016 fue Facebook (8\%), después de Fox News (19\%) y CNN (13\%). Facebook fue, por lo tanto, la principal fuente no televisiva citada por los partidarios de ambos candidatos presidenciales (Gottfried et al., 2017). En Estados Unidos, la penetración de Facebook es aún más fuerte entre los millennials cuando buscan información política: el 61\% cita a Facebook como su fuente principal (Mitchell et al., 2015). Bell (2016) constata esta evidencia al afirmar que las redes sociales han absorbido no únicamente el periodismo, lo han absorbido todo.

\section{El uso de otras redes en política}

Aunque el presente estudio se centra en los enlaces que comparten a través de Twitter los políticos y los periodistas, también es interesante observar si estos actores enlazan a sus seguidores con el resto de las redes sociales, principalmente con Facebook, Youtube e Instagram.

Sobre el uso que hacen de las redes los actores políticos, gran parte de la literatura trata sobre procesos electorales en los países del norte de Europa. Larsson estudió cómo usaron Facebook los líderes de los partidos noruegos durante la campaña electoral de 2013 y descubrió que las publicaciones más populares eran aquellas en las que los líderes del partido se hacían eco de los apoyos recibidos o lanzaban críticas a sus contrincantes o a los dirigentes de medios; la retroalimentación más común de los ciudadanos fueron los "me gusta", sin que se dieran comentarios ni se compartiera el contenido (Larsson, 2015). Los parlamentarios en Suecia y Noruega usan las redes principalmente como herramienta para una campaña permanente (Larsson, 2016). Sin embargo, en Dinamarca, los miembros del Parlamento mantienen conversaciones activas con los ciudadanos a través de Facebook, en contraste con la falta de conversación que, en general, se establece a través de Twitter. Sørensen concluye que "comparados 
con los de otros países, los parlamentarios daneses tienen un grado relativamente alto de participación en las conversaciones políticas con los ciudadanos en Facebook" (Sørensen, 2016, p. 664).

Al comparar Twitter e Instagram, Larsson (2017) concluyó que, en la campaña de las elecciones noruegas de 2015, los partidos pequeños hacían más uso de Twitter que los grandes, quienes hacían más uso de Instagram que los pequeños. En las elecciones de Suecia de 2014, Russmann y Svensson (2017) estudiaron el uso que los partidos hicieron de Instagram en la campaña, centrándose en el poder deliberativo de la red, y concluyeron nuevamente que la interacción con los usuarios fue escasa y el debate político a través de las imágenes de esta red, nulo.

Además de medir cuantitativamente la actividad, algunos estudios se detienen en el contenido de los mensajes de las cuentas de Facebook de los líderes políticos. Bronstein (2013) estudió el uso de esta red por parte de los dos candidatos de las elecciones presidenciales de 2012 en Estados Unidos y concluyó que su propósito principal era reforzar sus vínculos afectivos con sus seguidores. Lee y Campbell (2016) estudiaron los artículos políticos en Facebook en el Reino Unido desde septiembre de 2013 hasta las elecciones de mayo de 2015 y concluyeron también que, si bien la mayoría no recibe gran atención, esta práctica ayuda a los partidos a mantener sus vínculos con sus seguidores.

Lilleker et al. (2015), que comparan el papel de los nuevos medios en las estrategias de campaña de 68 partidos políticos de 12 países europeos, llegaron a la conclusión de que el estilo de campaña hipermediática se está convirtiendo en una característica común en Europa, ya que el uso de las redes está más presente en las democracias más jóvenes.

Halpern y Gibbs (2013) diseccionan el uso por parte del equipo de comunicación de la Casa Blanca de las plataformas Facebook y YouTube y explican que la cortesía es menor en YouTube. Atribuyen este factor al hecho de que esta es una red más anónima y desindividualizada que Facebook.

En Estados Unidos, Parmelee y Roman (2019) estudiaron las motivaciones de los usuarios para seguir a sus dirigentes políticos en Instagram. Tras encuestar a más de 300 usuarios, llegaron a la conclusión de que el motivo principal tiene que ver con la búsqueda de información y guías a la hora de justificar seguir a un político. Más al norte, en Canadá, Lalancette y Raynauld (2019) estudiaron el uso de Instagram por parte de Justin Trudeau y concluyeron que a través de esta red el primer ministro canadiense proyecta su vida personal para fomentar los valores y las ideas de su formación política, el Partido Liberal, a la vez que refuerza su estado de campaña permanente.

La mayoría de los estudios académicos sobre YouTube se centran en observar cómo esta red fomenta la deliberación pública y la participación de los ciudadanos en el debate político. Boyd (2014) analiza los diferentes marcos de participación de la población en la deliberación política por medio de los comentarios sobre los discursos de Obama. Berrocal et al. (2014) también estudian la información política producida por los usuarios de YouTube después de un discurso de la alcaldesa de Madrid en 2013, Ana Botella, y señalan las características de lo que llaman prosumtion of politaintment en Internet, esto es, un consumo masivo, pero con una reacción pasiva por parte de los usuarios.

Los diputados objeto de este estudio también lo han sido del estudio que publican Selva-Ruiz y Caro-Castaño (2017) sobre su uso de Instagram: concluyen que esa red social contribuye a crear en los usuarios la ilusión de cercanía y acceso a cuestiones personales e íntimas de los miembros del Congreso de la XII legislatura.

\section{Procedimientos Metodologícos}

Para realizar este estudio se ha recurrido a una herramienta construida ad hoc para observar la actividad en Twitter de diversos grupos de actores sociales mediante el Digital Trace Data Analysis: Contexto.es.

Por medio de Contexto.es, se han creado dos grupos de usuarios de Twitter: uno de diputados y otro de periodistas. El primero incluye a los miembros del Congreso durante la XII legislatura española, que empezó el 19 de julio de 2016 y terminó el 21 de mayo de 2019. El primer gobierno bajo aquella legislatura acabó abruptamente 
el 1 de junio de 2018, cuando triunfó una moción de censura presentada por el Partido Socialista Obrero Español (PSOE), de centro izquierda, y su candidato a presidente, Pedro Sánchez, obtuvo la mayoría absoluta de votos al reunir 180 apoyos en la cámara (la mayoría está en 176): a los votos de su partido se sumaron los de las formaciones Podemos (izquierda) y los partidos nacionalistas o regionalistas Partido Nacionalista Vasco, Esquerra Republicana de Catalunya, Partit Demòcrata Europeu Català, Compromís, Bildu y Nueva Canarias.

Se ha tomado esta fecha como la del inicio de la observación; la del final es la del día en que termina la legislatura al constituirse el congreso correspondiente a la XIII legislatura, el día 21 de mayo de 2019, en aplicación de los resultados obtenidos en las elecciones anticipadas del 28 de abril. La herramienta creada con Contexto. es monitoriza la actividad de las cuentas de Twitter de los miembros seleccionados para cada uno de los grupos creados.

En el grupo de los diputados hay 331 miembros, de los cuales 326 corresponden realmente a diputados presentes y activos en Twitter durante la legislatura estudiada. Las cinco cuentas restantes corresponden al Congreso (@Congreso_Es) y a las de los grupos del PSOE, el Partido Popular (PP), Podemos y Ciudadanos. El resto de los diputados, hasta los 350, no tienen cuenta en Twitter o, si la tienen, no hicieron uso de ella durante el periodo estudiado.

En el grupo de los periodistas hay 158 miembros, y consiste en una selección de los miembros de los cuadros directivos de los medios con más audiencia en España que tienen más seguidores en las redes sociales (Twitter) y participantes asiduos de las tertulias audiovisuales (TV y radio) con más audiencia en España que tienen más followers en medios 2.0 (Twitter). Para ello se han tenido en cuenta los datos de audiencia y difusión de los medios españoles de 2018 y 2019, publicados en el Estudio General de Medios elaborado por la Asociación para la Investigación de Medios de Comunicación y los datos que publica la Oficina de Justificación de la Difusión para los datos de audiencia de medios digitales (OJD Interactiva Medios Digitales, 2019).

Se han observado, pues, los dominios que los miembros de los dos grupos han compartido vía Twitter, teniendo en cuenta los siguientes aspectos: por un lado, los dominios compartidos por más miembros y los compartidos más veces, que no coinciden necesariamente. Luego se comparan los resultados de ambos grupos. Se han descartado los dominios compartidos por menos del 3\% de los miembros de cada grupo.

Los creadores de la herramienta Contexto.es han tenido en cuenta el rol de los bots en las redes sociales: tras un análisis previo de las interacciones realizadas en los perfiles de las cuentas estudiadas, se han descartado las sospechosas de no pertenecer a usuarios personales o institucionales, al no responder sus características a las propias del comportamiento humano en la red. Por tanto, se ha hecho el análisis con la garantía de que detrás de todas las cuentas monitorizadas hay perfiles fiables, con humanos que las gestionan y realizan las interacciones registradas, incompatibles con el funcionamiento de un bot.

\section{Resultados}

\section{Diputados}

La mayoría de los enlaces compartidos por los diputados son de medios de comunicación, tanto los compartidos por mayor número de ellos como los compartidos más veces. Ocupan los primeros lugares los medios convencionales, tanto periódicos tradicionales o nativos digitales como agencias o cadenas de radio y televisión (Tabla 1). El dominio compartido por más diputados es elpais.com, y el compartido más veces, elmundo. es. Es destacable que okdiario.com aparece en la posición 16a en la lista de dominios compartidos por más miembros del grupo (un 30\%) y, sin embargo, es el cuarto en la lista de los que más veces han sido compartidos, 1.552 veces. 
Tabla 1. Dominios compartidos por los diputados de la XII legislatura en España (junio 2018 - mayo 2019).

\begin{tabular}{|c|c|c|c|c|c|c|c|c|c|}
\hline \multicolumn{10}{|c|}{331 miembros } \\
\hline \multicolumn{5}{|c|}{ Ordenado por número de miembros que han visitado el dominio } & \multicolumn{5}{|c|}{ Ordenado por número de veces que se ha visitado el dominio } \\
\hline \multicolumn{2}{|c|}{ Dominios } & \multirow{2}{*}{$\begin{array}{c}\text { Miembros } \\
219\end{array}$} & \multirow{2}{*}{$\frac{\%}{66}$} & \multirow{2}{*}{$\frac{\text { Veces }}{2.574}$} & \multicolumn{2}{|r|}{ Dominios } & \multirow{2}{*}{$\frac{\text { Miembros }}{166}$} & \multirow{2}{*}{$\frac{\%}{50}$} & \multirow{2}{*}{$\frac{\text { Veces }}{3.239}$} \\
\hline 1 & elpais.com & & & & 1 & elmundo.es & & & \\
\hline 2 & youtube.com & 211 & 64 & 1.324 & 2 & elpais.com & 219 & 66 & 2.574 \\
\hline 3 & europapress.es & 171 & 52 & 855 & 3 & facebook.com & 129 & 39 & 2.536 \\
\hline 4 & elmundo.es & 166 & 50 & 3.239 & 4 & okdiario.com & 98 & 30 & 1.552 \\
\hline 5 & cadenaser.com & 159 & 48 & 609 & 5 & abc.es & 140 & 42 & 1.495 \\
\hline 6 & lavanguardia.com & 156 & 47 & 831 & 6 & elespanol.com & 110 & 33 & 1.348 \\
\hline 7 & eldiario.es & 144 & 44 & 588 & 7 & youtube.com & 211 & 64 & 1.324 \\
\hline 8 & abc.es & 140 & 42 & 1.495 & 8 & gppopular.es & 57 & 17 & 922 \\
\hline 9 & m.eldiario.es & 138 & 42 & 529 & 9 & europapress.es & 171 & 52 & 855 \\
\hline 10 & elconfidencial.com & 132 & 40 & 818 & 10 & lavanguardia.com & 156 & 47 & 831 \\
\hline 11 & facebook.com & 129 & 39 & 2.536 & 11 & elconfidencial.com & 132 & 40 & 818 \\
\hline 12 & pscp.tv & 112 & 34 & 285 & 12 & cadenaser.com & 159 & 48 & 609 \\
\hline 13 & elespanol.com & 110 & 33 & 1.348 & 13 & lector.kioskoymas.com & 35 & 11 & 601 \\
\hline 14 & publico.es & 104 & 31 & 374 & 14 & eldiario.es & 144 & 44 & 588 \\
\hline 15 & rtve.es & 102 & 31 & 294 & 15 & esdiario.com & 69 & 21 & 584 \\
\hline 16 & okdiario.com & 98 & 30 & 1.552 & 16 & m.eldiario.es & 138 & 42 & 529 \\
\hline 17 & elperiodico.com & 96 & 29 & 409 & 17 & tribunafeminista.elplural.com & 26 & 8 & 503 \\
\hline 18 & larazon.es & 90 & 27 & 470 & 18 & pp.es & 59 & 18 & 475 \\
\hline 19 & eleconomista.es & 86 & 26 & 390 & 19 & larazon.es & 90 & 27 & 470 \\
\hline 20 & instagram.com & 80 & 24 & 300 & 20 & elindependiente.com & 77 & 23 & 462 \\
\hline 21 & vozpopuli.com & 80 & 24 & 351 & 21 & elperiodico.com & 96 & 29 & 409 \\
\hline 22 & blogs.elconfidencial.com & 79 & 24 & 325 & 22 & elnacional.cat & 28 & 8 & 405 \\
\hline 23 & 20minutos.es & 78 & 24 & 154 & 23 & eleconomista.es & 86 & 26 & 390 \\
\hline 24 & cope.es & 78 & 24 & 269 & 24 & publico.es & 104 & 31 & 374 \\
\hline 25 & huffingtonpost.es & 78 & 24 & 234 & 25 & vozpopuli.com & 80 & 24 & 351 \\
\hline 26 & elindependiente.com & 77 & 23 & 462 & 26 & libertaddigital.com & 57 & 17 & 349 \\
\hline 27 & esdiario.com & 69 & 21 & 584 & 27 & lasprovincias.es & 44 & 13 & 346 \\
\hline 28 & google.es & 65 & 20 & 133 & 28 & blogs.elconfidencial.com & 79 & 24 & 325 \\
\hline 29 & lavozdegalicia.es & 62 & 19 & 325 & 29 & lavozdegalicia.es & 62 & 19 & 325 \\
\hline 30 & politica.elpais.com & 59 & 18 & 133 & 30 & diariosur.es & 48 & 15 & 316 \\
\hline 31 & pp.es & 59 & 18 & 475 & 31 & valenciaplaza.com & 36 & 11 & 314 \\
\hline 32 & expansion.com & 58 & 18 & 199 & 32 & instagram.com & 80 & 24 & 300 \\
\hline 33 & gppopular.es & 57 & 17 & 922 & 33 & rtve.es & 102 & 31 & 294 \\
\hline 34 & libertaddigital.com & 57 & 17 & 349 & 34 & pscp.tv & 112 & 34 & 285 \\
\hline 35 & congreso.es & 56 & 17 & 188 & 35 & rrss.abc.es & 52 & 16 & 285 \\
\hline 36 & elplural.com & 55 & 17 & 132 & 36 & ara.cat & 27 & 8 & 271 \\
\hline 37 & atresplayer.com & 54 & 16 & 168 & 37 & cope.es & 78 & 24 & 269 \\
\hline 38 & libremercado.com & 52 & 16 & 239 & 38 & ver.abc.es & 48 & 15 & 259 \\
\hline 39 & rrss.abc.es & 52 & 16 & 285 & 39 & naciodigital.cat & 27 & 8 & 240 \\
\hline 40 & diariodesevilla.es & 51 & 15 & 90 & 40 & libremercado.com & 52 & 16 & 239 \\
\hline 41 & sevilla.abc.es & 50 & 15 & 165 & 41 & cronicaglobal.elespanol.com & 47 & 14 & 239 \\
\hline 42 & diariosur.es & 48 & 15 & 316 & 42 & huffingtonpost.es & 78 & 24 & 234 \\
\hline 43 & ver.abc.es & 48 & 15 & 259 & 43 & ww.cope.es & 33 & 10 & 228 \\
\hline 44 & cronicaglobal.elespanol.com & 47 & 14 & 239 & 44 & vilaweb.cat & 21 & 6 & 213 \\
\hline 45 & infolibre.es & 46 & 14 & 142 & 45 & expansion.com & 58 & 18 & 199 \\
\hline 46 & lasprovincias.es & 44 & 13 & 346 & 46 & levante-emv.com & 39 & 12 & 195 \\
\hline 47 & confilegal.com & 42 & 13 & 174 & 47 & ccma.cat & 30 & 9 & 193 \\
\hline 48 & Irzn.es & 42 & 13 & 185 & 48 & lahoradigital.com & 11 & 3 & 191 \\
\hline 49 & lainformacion.com & 41 & 12 & 69 & 49 & blogs.publico.es & 36 & 11 & 189 \\
\hline
\end{tabular}


Tabla 1. Dominios compartidos por los diputados de la XII legislatura en España (junio 2018 - mayo 2019).

2 del 4

\begin{tabular}{|c|c|c|c|c|c|c|c|c|c|}
\hline \multicolumn{10}{|c|}{331 miembros } \\
\hline \multicolumn{5}{|c|}{ Ordenado por número de miembros que han visitado el dominio } & \multicolumn{5}{|c|}{ Ordenado por número de veces que se ha visitado el dominio } \\
\hline \multicolumn{2}{|r|}{ Dominios } & \multirow{2}{*}{$\begin{array}{c}\text { Miembros } \\
41\end{array}$} & \multirow{2}{*}{$\begin{array}{l}\% \\
12\end{array}$} & \multirow{2}{*}{$\frac{\text { Veces }}{65}$} & \multicolumn{2}{|r|}{ Dominios } & \multirow{2}{*}{$\frac{\text { Miembros }}{56}$} & \multirow{2}{*}{$\frac{\%}{17}$} & \multirow{2}{*}{$\frac{\text { Veces }}{188}$} \\
\hline 50 & m.europapress.es & & & & 50 & congreso.es & & & \\
\hline 51 & theobjective.com & 41 & 12 & 75 & 51 & Irzn.es & 42 & 13 & 185 \\
\hline 52 & levante-emv.com & 39 & 12 & 195 & 52 & diarioinformacion.com & 31 & 9 & 181 \\
\hline 53 & amp.elmundo.es & 37 & 11 & 62 & 53 & confilegal.com & 42 & 13 & 174 \\
\hline 54 & laverdad.es & 37 & 11 & 150 & 54 & atresplayer.com & 54 & 16 & 168 \\
\hline 55 & ondacero.es & 37 & 11 & 60 & 55 & sevilla.abc.es & 50 & 15 & 165 \\
\hline 56 & blogs.publico.es & 36 & 11 & 189 & 56 & 20minutos.es & 78 & 24 & 154 \\
\hline 57 & valenciaplaza.com & 36 & 11 & 314 & 57 & participa.podemos.info & 25 & 8 & 154 \\
\hline 58 & lector.kioskoymas.com & 35 & 11 & 601 & 58 & elmon.cat & 19 & 6 & 151 \\
\hline 59 & cincodias.elpais.com & 34 & 10 & 80 & 59 & laverdad.es & 37 & 11 & 150 \\
\hline 60 & m.youtube.com & 34 & 10 & 53 & 60 & infolibre.es & 46 & 14 & 142 \\
\hline 61 & ctxt.es & 33 & 10 & 72 & 61 & google.es & 65 & 20 & 133 \\
\hline 62 & ww.cope.es & 33 & 10 & 228 & 62 & politica.elpais.com & 59 & 18 & 133 \\
\hline 63 & antena3.com & 32 & 10 & 63 & 63 & elplural.com & 55 & 17 & 132 \\
\hline 64 & cuartopoder.es & 32 & 10 & 71 & 64 & laregion.es & 10 & 3 & 120 \\
\hline 65 & telecinco.es & 32 & 10 & 61 & 65 & fundacionsistema.com & 15 & 5 & 107 \\
\hline 66 & diarioinformacion.com & 31 & 9 & 181 & 66 & elpuntavui.cat & 21 & 6 & 102 \\
\hline 67 & theguardian.com & 31 & 9 & 56 & 67 & elcatalan.es & 16 & 5 & 97 \\
\hline 68 & ccma.cat & 30 & 9 & 193 & 68 & diariocordoba.com & 17 & 5 & 95 \\
\hline 69 & elsiglodeuropa.es & 29 & 9 & 42 & 69 & diariodesevilla.es & 51 & 15 & 90 \\
\hline 70 & m.publico.es & 29 & 9 & 52 & 70 & elperiodicomediterraneo.com & 9 & 3 & 84 \\
\hline 71 & abcblogs.abc.es & 28 & 8 & 57 & 71 & lamoncloa.gob.es & 16 & 5 & 82 \\
\hline 72 & elconfidencialdigital.com & 28 & 8 & 45 & 72 & cincodias.elpais.com & 34 & 10 & 80 \\
\hline 73 & elnacional.cat & 28 & 8 & 405 & 73 & periodistadigital.com & 24 & 7 & 80 \\
\hline 74 & ara.cat & 27 & 8 & 271 & 74 & eitb.eus & 20 & 6 & 79 \\
\hline 75 & casareal.es & 27 & 8 & 49 & 75 & theobjective.com & 41 & 12 & 75 \\
\hline 76 & diario16.com & 27 & 8 & 67 & 76 & ctxt.es & 33 & 10 & 72 \\
\hline 77 & naciodigital.cat & 27 & 8 & 240 & 77 & cuartopoder.es & 32 & 10 & 71 \\
\hline 78 & change.org & 26 & 8 & 33 & 78 & eldiestro.es & 15 & 5 & 70 \\
\hline 79 & elsaltodiario.com & 26 & 8 & 56 & 79 & lainformacion.com & 41 & 12 & 69 \\
\hline 80 & play.cadenaser.com & 26 & 8 & 40 & 80 & nuevatribuna.es & 23 & 7 & 69 \\
\hline 81 & psoe.es & 26 & 8 & 68 & 81 & psoe.es & 26 & 8 & 68 \\
\hline 82 & tribunafeminista.elplural.com & 26 & 8 & 503 & 82 & redaccionmedica.com & 22 & 7 & 68 \\
\hline 83 & diariovasco.com & 25 & 8 & 35 & 83 & diaridegirona.cat & 12 & 4 & 68 \\
\hline 84 & participa.podemos.info & 25 & 8 & 154 & 84 & diario16.com & 27 & 8 & 67 \\
\hline 85 & andaluciainformacion.es & 24 & 7 & 39 & 85 & m.europapress.es & 41 & 12 & 65 \\
\hline 86 & periodistadigital.com & 24 & 7 & 80 & 86 & socialistes.cat & 9 & 3 & 65 \\
\hline 87 & elcorreo.com & 23 & 7 & 34 & 87 & antena3.com & 32 & 10 & 63 \\
\hline 88 & nuevatribuna.es & 23 & 7 & 69 & 88 & farodevigo.es & 18 & 5 & 63 \\
\hline 89 & politico.eu & 22 & 7 & 31 & 89 & lavoz.gal & 11 & 3 & 63 \\
\hline 90 & redaccionmedica.com & 22 & 7 & 68 & 90 & amp.elmundo.es & 37 & 11 & 62 \\
\hline 91 & servimedia.es & 22 & 7 & 36 & 91 & telecinco.es & 32 & 10 & 61 \\
\hline 92 & alicanteplaza.es & 21 & 6 & 52 & 92 & hoy.es & 15 & 5 & 61 \\
\hline 93 & amp.europapress.es & 21 & 6 & 28 & 93 & open.spotify.com & 15 & 5 & 61 \\
\hline 94 & eldia.es & 21 & 6 & 26 & 94 & ondacero.es & 37 & 11 & 60 \\
\hline 95 & elperiodi.co & 21 & 6 & 38 & 95 & laopiniondemalaga.es & 17 & 5 & 60 \\
\hline 96 & elpuntavui.cat & 21 & 6 & 102 & 96 & lapaseata.net & 12 & 4 & 60 \\
\hline 97 & heraldo.es & 21 & 6 & 51 & 97 & abcblogs.abc.es & 28 & 8 & 57 \\
\hline 98 & ondace.ro & 21 & 6 & 29 & 98 & eltemps.cat & 11 & 3 & 57 \\
\hline
\end{tabular}


Tabla 1. Dominios compartidos por los diputados de la XII legislatura en España (junio 2018 - mayo 2019).

\begin{tabular}{|c|c|c|c|c|c|c|c|c|}
\hline \multicolumn{9}{|c|}{331 miembros } \\
\hline \multicolumn{4}{|c|}{ Ordenado por número de miembros que han visitado el dominio } & \multicolumn{5}{|c|}{ Ordenado por número de veces que se ha visitado el dominio } \\
\hline Dominios & Miembros & $\%$ & Veces & & Dominios & Miembros & $\%$ & Veces \\
\hline vilaweb.cat & 21 & 6 & 213 & 99 & theguardian.com & 31 & 9 & 56 \\
\hline eitb.eus & 20 & 6 & 79 & 100 & elsaltodiario.com & 26 & 8 & 56 \\
\hline pp100dias.pp.es & 20 & 6 & 20 & 101 & m.youtube.com & 34 & 10 & 53 \\
\hline elmon.cat & 19 & 6 & 151 & 102 & m.publico.es & 29 & 9 & 52 \\
\hline espana.abc.es & 19 & 6 & 26 & 103 & alicanteplaza.es & 21 & 6 & 52 \\
\hline leonoticias.com & 19 & 6 & 38 & 104 & heraldo.es & 21 & 6 & 51 \\
\hline elnortedecastilla.es & 18 & 5 & 44 & 105 & casareal.es & 27 & 8 & 49 \\
\hline farodevigo.es & 18 & 5 & 63 & 106 & consalud.es & 16 & 5 & 48 \\
\hline lainfo.news & 18 & 5 & 22 & 107 & laopiniondemurcia.es & 15 & 5 & 48 \\
\hline prensa.empleo.gob.es & 18 & 5 & 18 & 108 & elprogreso.es & 13 & 4 & 48 \\
\hline SS.MM & 18 & 5 & 27 & 109 & ppcv.com & 10 & 3 & 47 \\
\hline ciudadanos-cs.org & 17 & 5 & 42 & 110 & elconfidencialdigital.com & 28 & 8 & 45 \\
\hline diariocordoba.com & 17 & 5 & 95 & 111 & elnortedecastilla.es & 18 & 5 & 44 \\
\hline laopiniondemalaga.es & 17 & 5 & 60 & 112 & elsiglodeuropa.es & 29 & 9 & 42 \\
\hline Ine.es & 17 & 5 & 28 & 113 & ciudadanos-cs.org & 17 & 5 & 42 \\
\hline consalud.es & 16 & 5 & 48 & 114 & elperiodicodearagon.com & 10 & 3 & 41 \\
\hline elcatalan.es & 16 & 5 & 97 & 115 & play.cadenaser.com & 26 & 8 & 40 \\
\hline lamoncloa.gob.es & 16 & 5 & 82 & 116 & andaluciainformacion.es & 24 & 7 & 39 \\
\hline moncloa.com & 16 & 5 & 23 & 117 & diaridetarragona.com & 13 & 4 & 39 \\
\hline nytimes.com & 16 & 5 & 27 & 118 & elperiodi.co & 21 & 6 & 38 \\
\hline tv.libertaddigital.com & 16 & 5 & 19 & 119 & leonoticias.com & 19 & 6 & 38 \\
\hline agendapublica.elperiodico.com & 15 & 5 & 37 & 120 & economist.com & 10 & 3 & 38 \\
\hline app.congreso.es & 15 & 5 & 19 & 121 & mas.laopiniondemalaga.es & 10 & 3 & 38 \\
\hline eldiestro.es & 15 & 5 & 70 & 122 & agendapublica.elperiodico.com & 15 & 5 & 37 \\
\hline fundacionsistema.com & 15 & 5 & 107 & 123 & servimedia.es & 22 & 7 & 36 \\
\hline hoy.es & 15 & 5 & 61 & 124 & autonomosyemprendedor.es & 12 & 4 & 36 \\
\hline intereconomia.com & 15 & 5 & 20 & 125 & diariovasco.com & 25 & 8 & 35 \\
\hline laopiniondemurcia.es & 15 & 5 & 48 & 126 & eldigitalcastillalamancha.es & 10 & 3 & 35 \\
\hline letraslibres.com & 15 & 5 & 26 & 127 & gaceta.es & 10 & 3 & 35 \\
\hline marca.com & 15 & 5 & 17 & 128 & elcorreo.com & 23 & 7 & 34 \\
\hline open.spotify.com & 15 & 5 & 61 & 129 & change.org & 26 & 8 & 33 \\
\hline t.me & 15 & 5 & 30 & 130 & navarra.elespanol.com & 10 & 3 & 33 \\
\hline telemadrid.es & 15 & 5 & 20 & 131 & ivoox.com & 10 & 3 & 32 \\
\hline elperiodico.cat & 14 & 4 & 30 & 132 & politico.eu & 22 & 7 & 31 \\
\hline fundacionemprendedores.com & 14 & 4 & 14 & 133 & t.me & 15 & 5 & 30 \\
\hline m.ara.cat & 14 & 4 & 22 & 134 & elperiodico.cat & 14 & 4 & 30 \\
\hline boe.es & 13 & 4 & 14 & 135 & elperiodicodeaqui.com & 10 & 3 & 30 \\
\hline diaridetarragona.com & 13 & 4 & 39 & 136 & ondace.ro & 21 & 6 & 29 \\
\hline elprogreso.es & 13 & 4 & 48 & 137 & amp.europapress.es & 21 & 6 & 28 \\
\hline lavozdealmeria.com & 13 & 4 & 20 & 138 & Ine.es & 17 & 5 & 28 \\
\hline rsocial.elmundo.orbyt.es & 13 & 4 & 17 & 139 & diariodecadiz.es & 9 & 3 & 28 \\
\hline autonomosyemprendedor.es & 12 & 4 & 36 & 140 & SS.MM & 18 & 5 & 27 \\
\hline bbc.com & 12 & 4 & 18 & 141 & nytimes.com & 16 & 5 & 27 \\
\hline cuatro.com & 12 & 4 & 13 & 142 & eldia.es & 21 & 6 & 26 \\
\hline diaridegirona.cat & 12 & 4 & 68 & 143 & espana.abc.es & 19 & 6 & 26 \\
\hline diariodeavisos.elespanol.com & 12 & 4 & 16 & 144 & letraslibres.com & 15 & 5 & 26 \\
\hline ecodiario.eleconomista.es & 12 & 4 & 17 & 145 & elperiodic.com & 10 & 3 & 26 \\
\hline elcorreogallego.es & 12 & 4 & 23 & 146 & diariodenavarra.es & 11 & 3 & 24 \\
\hline ft.com & 12 & 4 & 15 & 147 & moncloa.com & 16 & 5 & 23 \\
\hline hayderecho.com & 12 & 4 & 13 & 148 & elcorreogallego.es & 12 & 4 & 23 \\
\hline
\end{tabular}


Tabla 1. Dominios compartidos por los diputados de la XII legislatura en España (junio 2018 - mayo 2019).

4 del 4

\begin{tabular}{|c|c|c|c|c|c|c|c|c|c|}
\hline \multicolumn{10}{|c|}{331 miembros } \\
\hline \multicolumn{5}{|c|}{ Ordenado por número de miembros que han visitado el dominio } & \multicolumn{5}{|c|}{ Ordenado por número de veces que se ha visitado el dominio } \\
\hline \multicolumn{2}{|c|}{ Dominios } & \multirow{2}{*}{$\frac{\text { Miembros }}{12}$} & \multirow{2}{*}{$\frac{\%}{4}$} & \multirow{2}{*}{$\frac{\text { Veces }}{60}$} & \multicolumn{2}{|r|}{ Dominios } & \multirow{2}{*}{$\frac{\text { Miembros }}{10}$} & \multirow{2}{*}{$\frac{\%}{3}$} & \multirow{2}{*}{$\frac{\text { Veces }}{23}$} \\
\hline 149 & lapaseata.net & & & & 149 & elperiodicoextremadura.com & & & \\
\hline 150 & lavozdeasturias.es & 12 & 4 & 15 & 150 & encastillalamancha.es & 9 & 3 & 23 \\
\hline 151 & m.elconfidencialdigital.com & 12 & 4 & 13 & 151 & sevillainfo.es & 9 & 3 & 23 \\
\hline 152 & politica.e-noticies.es & 12 & 4 & 21 & 152 & lainfo.news & 18 & 5 & 22 \\
\hline 153 & verne.elpais.com & 12 & 4 & 15 & 153 & m.ara.cat & 14 & 4 & 22 \\
\hline 154 & bolsamania.com & 11 & 3 & 14 & 154 & dolcacatalunya.com & 10 & 3 & 22 \\
\hline 155 & canarias7.es & 11 & 3 & 18 & 155 & politica.e-noticies.es & 12 & 4 & 21 \\
\hline 156 & diariodenavarra.es & 11 & 3 & 24 & 156 & laopinioncoruna.es & 10 & 3 & 21 \\
\hline 157 & eltemps.cat & 11 & 3 & 57 & 157 & pp100dias.pp.es & 20 & 6 & 20 \\
\hline 158 & lahoradigital.com & 11 & 3 & 191 & 158 & intereconomia.com & 15 & 5 & 20 \\
\hline 159 & lavoz.gal & 11 & 3 & 63 & 159 & telemadrid.es & 15 & 5 & 20 \\
\hline 160 & navarth.blogspot.com & 11 & 3 & 20 & 160 & lavozdealmeria.com & 13 & 4 & 20 \\
\hline 161 & rac1.cat & 11 & 3 & 17 & 161 & navarth.blogspot.com & 11 & 3 & 20 \\
\hline 162 & cat.elpais.com & 10 & 3 & 15 & 162 & tv.libertaddigital.com & 16 & 5 & 19 \\
\hline 163 & clm24.es & 10 & 3 & 16 & 163 & app.congreso.es & 15 & 5 & 19 \\
\hline 164 & crtvg.es & 10 & 3 & 15 & 164 & elcomercio.es & 10 & 3 & 19 \\
\hline 165 & dolcacatalunya.com & 10 & 3 & 22 & 165 & prensa.empleo.gob.es & 18 & 5 & 18 \\
\hline 166 & economist.com & 10 & 3 & 38 & 166 & bbc.com & 12 & 4 & 18 \\
\hline 167 & elcomercio.es & 10 & 3 & 19 & 167 & canarias7.es & 11 & 3 & 18 \\
\hline 168 & eldigitalcastillalamancha.es & 10 & 3 & 35 & 168 & go.shr.lc & 10 & 3 & 18 \\
\hline 169 & elperiodic.com & 10 & 3 & 26 & 169 & marca.com & 15 & 5 & 17 \\
\hline 170 & elperiodicodeaqui.com & 10 & 3 & 30 & 170 & rsocial.elmundo.orbyt.es & 13 & 4 & 17 \\
\hline 171 & elperiodicodearagon.com & 10 & 3 & 41 & 171 & ecodiario.eleconomista.es & 12 & 4 & 17 \\
\hline 172 & elperiodicoextremadura.com & 10 & 3 & 23 & 172 & rac1.cat & 11 & 3 & 17 \\
\hline 173 & gaceta.es & 10 & 3 & 35 & 173 & diariodeavisos.elespanol.com & 12 & 4 & 16 \\
\hline 174 & go.shr.lc & 10 & 3 & 18 & 174 & clm24.es & 10 & 3 & 16 \\
\hline 175 & ivoox.com & 10 & 3 & 32 & 175 & ft.com & 12 & 4 & 15 \\
\hline 176 & laopinioncoruna.es & 10 & 3 & 21 & 176 & lavozdeasturias.es & 12 & 4 & 15 \\
\hline 177 & laregion.es & 10 & 3 & 120 & 177 & verne.elpais.com & 12 & 4 & 15 \\
\hline 178 & mas.laopiniondemalaga.es & 10 & 3 & 38 & 178 & cat.elpais.com & 10 & 3 & 15 \\
\hline 179 & navarra.elespanol.com & 10 & 3 & 33 & 179 & crtvg.es & 10 & 3 & 15 \\
\hline 180 & ppcv.com & 10 & 3 & 47 & 180 & cnn.it & 9 & 3 & 15 \\
\hline 181 & yovoy.pp.es & 10 & 3 & 12 & 181 & diariofarma.com & 9 & 3 & 15 \\
\hline 182 & cnn.it & 9 & 3 & 15 & 182 & fundacionemprendedores.com & 14 & 4 & 14 \\
\hline 183 & diariodecadiz.es & 9 & 3 & 28 & 183 & boe.es & 13 & 4 & 14 \\
\hline 184 & diariodemallorca.es & 9 & 3 & 13 & 184 & bolsamania.com & 11 & 3 & 14 \\
\hline 185 & diariofarma.com & 9 & 3 & 15 & 185 & cuatro.com & 12 & 4 & 13 \\
\hline 186 & dozz.es & 9 & 3 & 9 & 186 & hayderecho.com & 12 & 4 & 13 \\
\hline 187 & economiadigital.es & 9 & 3 & 11 & 187 & m.elconfidencialdigital.com & 12 & 4 & 13 \\
\hline 188 & electomania.es & 9 & 3 & 10 & 188 & diariodemallorca.es & 9 & 3 & 13 \\
\hline 189 & elperiodicomediterraneo.com & 9 & 3 & 84 & 189 & lamarea.com & 9 & 3 & 13 \\
\hline 190 & encastillalamancha.es & 9 & 3 & 23 & 190 & vertele.eldiario.es & 9 & 3 & 13 \\
\hline 191 & europapress.tv & 9 & 3 & 9 & 191 & vimeo.com & 9 & 3 & 13 \\
\hline 192 & lamarea.com & 9 & 3 & 13 & 192 & yovoy.pp.es & 10 & 3 & 12 \\
\hline 193 & podemos.info & 9 & 3 & 12 & 193 & podemos.info & 9 & 3 & 12 \\
\hline 194 & sevillainfo.es & 9 & 3 & 23 & 194 & economiadigital.es & 9 & 3 & 11 \\
\hline 195 & socialistes.cat & 9 & 3 & 65 & 195 & electomania.es & 9 & 3 & 10 \\
\hline 196 & ultimahora.es & 9 & 3 & 10 & 196 & ultimahora.es & 9 & 3 & 10 \\
\hline 197 & vertele.eldiario.es & 9 & 3 & 13 & 197 & dozz.es & 9 & 3 & 9 \\
\hline 198 & vimeo.com & 9 & 3 & 13 & 198 & europapress.tv & 9 & 3 & 9 \\
\hline Total & & & & & & & & & 37.992 \\
\hline
\end{tabular}

Fuente: Elaboración propia (2020). 
En cuanto a las redes sociales, YouTube es el segundo dominio compartido por más miembros y el sexto más veces compartido. Facebook da unos resultados similares: no en cuanto al número de miembros que comparten el dominio, pero sí a las veces: es el tercer dominio más veces compartido y el $11^{\circ}$ compartido por más miembros. Instagram, sin embargo, aparece mucho más abajo en ambas listas: en la posición 20a y 32a respectivamente.

No se observan diferencias destacables en cuanto a la difusión de los dominios de los medios nativos digitales entre los compartidos por más miembros y los compartidos más veces: Eldiario.es, elconfidencial.com, elespanol.com, publico.es y okdiario.com encabezan por este orden los sitios web compartidos por más miembros, y los cuatro compartidos más veces son, también por orden, okdiario.com, elespanol.com, elconfidencial.com y el diario.es. La web de publico.es, sin embargo, sí aparece en posición más alejada (la 24a) en la lista de las webs más veces compartidas.

Los enlaces a las pocas webs de grupos, partidos o entidades políticas y a instituciones no aparecen en los primeros lugares de las listas, aunque es destacable que los dominios vinculados al Partido Popular reflejan mucha más actividad que los del resto de formaciones: el dominio pp.es es el compartido por más miembros, en posición 31a y el dominio gppopular.es le sigue en la posición 33a. También son los compartidos más veces: el gppopular.es está en octava posición y pp.es en posición 18a. Es notable observar que los dominios vinculados a los otros tres principales partidos de la cámara, PSOE, Ciudadanos y Podemos, son mucho menos compartidos que los del PP, tanto en número de miembros que los comparten como en veces compartidos. En cuanto al resto de las instituciones, la presencia de los dominios es muy poco relevante: el enlace a Congreso.es está en posición 35ª en el ranking de los miembros que lo han compartido y en la 50a en el de las veces compartido. Lo mismo sucede con la Casa Real o la Moncloa (sede oficial del presidente del gobierno de España), compartidos por pocos usuarios y en muy pocas ocasiones.

Los miembros del Congreso han compartido 198 enlaces distintos un total de 37.992 veces en el periodo estudiado. Las veces que esta compartición no ha sido a un medio de comunicación o a una red social como Youtube, Facebook o Instagram suman 2.325, esto es un 6,1\%. Por tanto, en el 93,9\% de las veces que un enlace ha sido difundido a través de su cuenta de Twitter, lo era a la web de un medio.

Los medios a los que se enlaza son prácticamente en su totalidad españoles: los únicos medios extranjeros a los que se enlaza (en muy pocas ocasiones) son: The Guardian (31 miembros un total de 57 veces), Politico (22 miembros, 31 veces), New York Times (16 y 27), The Economist (10 y 38), BBC (12 y 18), Financial Times (12 y 15) y CNN (9 y 15).

\section{Periodistas}

El dominio compartido por más periodistas en sus cuentas de Twitter es el del diario El País. El resto de los periódicos convencionales también está entre las primeras posiciones en cuanto al número de miembros que los comparten (Tabla 2). Son, por este orden: El Mundo, La Vanguardia, El Periódico y ABC, que ocupan las posiciones 3 a la 6. De toda la lista de 188 webs compartidas por más periodistas, prácticamente todas son a medios de comunicación, tales como periódicos, agencias, cadenas de radio o televisión o medios nativos digitales. En cuanto a estos últimos, cabe decir que los compartidos por más miembros son, por este orden, elconfidencial. com, elespanol.com y eldiario.es, que están en las posiciones 7a, 9a y 10ª. Sin embargo, a la hora de observar el número de veces que los dominios han sido compartidos, aparece una paradoja: el medio más veces compartido es periodistadigital.com, un total de 10.505 veces (un 17,5\%), a pesar de que solo lo han compartido 8 de los 158 miembros del grupo. Tras él aparecen sevilla.abc.es, eldiario.es y elmundo.es. Pero en quinta posición aparece vilaweb.cat, un medio digital nativo catalán que ofrece todos sus contenidos exclusivamente en catalán y en inglés, no en español. Sin embargo, fue compartido 2.725 veces por solo 8 miembros del grupo de periodistas. Otro dato llamativo es que el dominio larazon.es aparece en el lugar 66, compartido solo por 9 miembros 82 veces; sin embargo, el dominio Irzn.es, vinculado al mismo periódico, aparece compartido solo por seis miembros, en la posición 105a, pero compartido 2.153 veces, lo que lo sitúa en el séptimo lugar de los más veces compartidos. 
Tabla 2. Dominios compartidos por periodistas en España (junio 2018 - mayo 2019).

1 del 4

\begin{tabular}{|c|c|c|c|c|c|c|c|c|c|}
\hline \multicolumn{10}{|c|}{158 miembros } \\
\hline \multicolumn{5}{|c|}{ Ordenado por número de miembros que han visitado el dominio } & \multicolumn{5}{|c|}{ Ordenado por número de veces que se ha visitado el dominio } \\
\hline \multicolumn{2}{|c|}{ Dominios } & \multirow{2}{*}{$\frac{\text { Miembros }}{90}$} & \multirow{2}{*}{$\begin{array}{l}\% \\
57\end{array}$} & \multirow{2}{*}{$\begin{array}{l}\text { Veces } \\
2.273\end{array}$} & \multicolumn{2}{|r|}{ Dominios } & \multirow{2}{*}{$\frac{\text { Miembros }}{8}$} & \multirow{2}{*}{$\frac{\%}{5}$} & \multirow{2}{*}{$\frac{\text { Veces }}{10.505}$} \\
\hline 1 & elpais.com & & & & 1 & periodistadigital.com & & & \\
\hline 2 & youtube.com & 78 & 49 & 559 & 2 & sevilla.abc.es & 15 & 9 & 6.234 \\
\hline 3 & elmundo.es & 68 & 43 & 3.467 & 3 & eldiario.es & 39 & 25 & 5.877 \\
\hline 4 & lavanguardia.com & 60 & 38 & 611 & 4 & elmundo.es & 68 & 43 & 3.467 \\
\hline 5 & elperiodico.com & 46 & 29 & 1.001 & 5 & vilaweb.cat & 8 & 5 & 2.725 \\
\hline 6 & abc.es & 45 & 28 & 1.425 & 6 & elpais.com & 90 & 57 & 2.273 \\
\hline 7 & elconfidencial.com & 44 & 28 & 1.817 & 7 & Irzn.es & 6 & 4 & 2.153 \\
\hline 8 & instagram.com & 44 & 28 & 543 & 8 & elconfidencial.com & 44 & 28 & 1.817 \\
\hline 9 & elespanol.com & 41 & 26 & 658 & 9 & cronicaglobal.elespanol.com & 18 & 11 & 1.696 \\
\hline 10 & eldiario.es & 39 & 25 & 5.877 & 10 & elindependiente.com & 31 & 20 & 1.467 \\
\hline 11 & blogs.elconfidencial.com & 36 & 23 & 314 & 11 & abc.es & 45 & 28 & 1.425 \\
\hline 12 & cadenaser.com & 35 & 22 & 147 & 12 & elnortedecastilla.es & 9 & 6 & 1.328 \\
\hline 13 & m.eldiario.es & 35 & 22 & 1.199 & 13 & m.eldiario.es & 35 & 22 & 1.199 \\
\hline 14 & nytimes.com & 34 & 22 & 315 & 14 & diariodecadiz.es & 7 & 4 & 1.127 \\
\hline 15 & elindependiente.com & 31 & 20 & 1.467 & 15 & elperiodico.com & 46 & 29 & 1.001 \\
\hline 16 & vozpopuli.com & 31 & 20 & 434 & 16 & ara.cat & 10 & 6 & 893 \\
\hline 17 & theguardian.com & 30 & 19 & 114 & 17 & expansion.com & 23 & 15 & 830 \\
\hline 18 & politica.elpais.com & 28 & 18 & 75 & 18 & elconfidencialdigital.com & 11 & 7 & 733 \\
\hline 19 & efe.com & 23 & 15 & 71 & 19 & infolibre.es & 16 & 10 & 664 \\
\hline 20 & expansion.com & 23 & 15 & 830 & 20 & elespanol.com & 41 & 26 & 658 \\
\hline 21 & rtve.es & 23 & 15 & 43 & 21 & burgosconecta.es & 4 & 3 & 639 \\
\hline 22 & huffingtonpost.es & 22 & 14 & 433 & 22 & lasexta.com & 15 & 9 & 614 \\
\hline 23 & pscp.tv & 21 & 13 & 179 & 23 & lavanguardia.com & 60 & 38 & 611 \\
\hline 24 & europapress.es & 19 & 12 & 29 & 24 & youtube.com & 78 & 49 & 559 \\
\hline 25 & publico.es & 19 & 12 & 171 & 25 & instagram.com & 44 & 28 & 543 \\
\hline 26 & cronicaglobal.elespanol.com & 18 & 11 & 1.696 & 26 & vozpopuli.com & 31 & 20 & 434 \\
\hline 27 & elmundotoday.com & 18 & 11 & 37 & 27 & huffingtonpost.es & 22 & 14 & 433 \\
\hline 28 & google.es & 18 & 11 & 30 & 28 & as.com & 9 & 6 & 429 \\
\hline 29 & washingtonpost.com & 18 & 11 & 72 & 29 & rrss.abc.es & 14 & 9 & 364 \\
\hline 30 & facebook.com & 17 & 11 & 51 & 30 & antena3.com & 10 & 6 & 343 \\
\hline 31 & ft.com & 17 & 11 & 35 & 31 & ver.abc.es & 12 & 8 & 326 \\
\hline 32 & infolibre.es & 16 & 10 & 664 & 32 & laverdad.es & 9 & 6 & 321 \\
\hline 33 & lainformacion.com & 16 & 10 & 62 & 33 & nytimes.com & 34 & 22 & 315 \\
\hline 34 & linkedin.com & 16 & 10 & 76 & 34 & blogs.elconfidencial.com & 36 & 23 & 314 \\
\hline 35 & bbc.com & 15 & 9 & 43 & 35 & newtral.es & 5 & 3 & 313 \\
\hline 36 & ctxt.es & 15 & 9 & 66 & 36 & eldiariomontanes.es & 4 & 3 & 302 \\
\hline 37 & lasexta.com & 15 & 9 & 614 & 37 & diariosur.es & 13 & 8 & 249 \\
\hline 38 & medium.com & 15 & 9 & 33 & 38 & servimedia.es & 4 & 3 & 241 \\
\hline 39 & sevilla.abc.es & 15 & 9 & 6.234 & 39 & elcorreo.com & 14 & 9 & 209 \\
\hline 40 & cnn.it & 14 & 9 & 25 & 40 & diariodesevilla.es & 12 & 8 & 194 \\
\hline 41 & elcorreo.com & 14 & 9 & 209 & 41 & pscp.tv & 21 & 13 & 179 \\
\hline 42 & rrss.abc.es & 14 & 9 & 364 & 42 & vertele.eldiario.es & 14 & 9 & 172 \\
\hline 43 & vertele.eldiario.es & 14 & 9 & 172 & 43 & publico.es & 19 & 12 & 171 \\
\hline 44 & zendalibros.com & 14 & 9 & 60 & 44 & lector.kioskoymas.com & 11 & 7 & 162 \\
\hline 45 & amp.elmundo.es & 13 & 8 & 31 & 45 & elperiodi.co & 10 & 6 & 157 \\
\hline 46 & cincodias.elpais.com & 13 & 8 & 16 & 46 & cadenaser.com & 35 & 22 & 147 \\
\hline 47 & diariosur.es & 13 & 8 & 249 & 47 & theguardian.com & 30 & 19 & 114 \\
\hline 48 & diariodesevilla.es & 12 & 8 & 194 & 48 & dozz.es & 7 & 4 & 112 \\
\hline 49 & lavozdegalicia.es & 12 & 8 & 14 & 49 & elpuntavui.cat & 4 & 3 & 111 \\
\hline 50 & reuters.com & 12 & 8 & 32 & 50 & abcblogs.abc.es & 6 & 4 & 84 \\
\hline 51 & ver.abc.es & 12 & 8 & 326 & 51 & larazon.es & 9 & 6 & 82 \\
\hline 52 & verne.elpais.com & 12 & 8 & 13 & 52 & lasprovincias.es & 9 & 6 & 80 \\
\hline 53 & cope.es & 11 & 7 & 70 & 53 & ondace.ro & 6 & 4 & 79 \\
\hline
\end{tabular}


Tabla 2. Dominios compartidos por periodistas en España (junio 2018 - mayo 2019).

\begin{tabular}{|c|c|c|c|c|c|c|c|c|c|}
\hline \multicolumn{10}{|c|}{158 miembros } \\
\hline \multicolumn{5}{|c|}{ Ordenado por número de miembros que han visitado el dominio } & \multicolumn{5}{|c|}{ Ordenado por número de veces que se ha visitado el dominio } \\
\hline \multicolumn{2}{|c|}{ Dominios } & \multirow{2}{*}{$\begin{array}{c}\text { Miembros } \\
11\end{array}$} & \multirow{2}{*}{$\frac{\%}{7}$} & \multirow{2}{*}{$\frac{\text { Veces }}{733}$} & \multicolumn{2}{|r|}{ Dominios } & \multirow{2}{*}{$\begin{array}{c}\text { Miembros } \\
16\end{array}$} & \multirow{2}{*}{$\frac{\%}{10}$} & \multirow{2}{*}{$\frac{\text { Veces }}{76}$} \\
\hline 54 & elconfidencialdigital.com & & & & 54 & linkedin.com & & & \\
\hline 55 & lector.kioskoymas.com & 11 & 7 & 162 & 55 & hoy.es & 4 & 3 & 76 \\
\hline 56 & okdiario.com & 11 & 7 & 22 & 56 & politica.elpais.com & 28 & 18 & 75 \\
\hline 57 & antena3.com & 10 & 6 & 343 & 57 & lavozdigital.es & 5 & 3 & 75 \\
\hline 58 & apmadrid.es & 10 & 6 & 27 & 58 & washingtonpost.com & 18 & 11 & 72 \\
\hline 59 & ara.cat & 10 & 6 & 893 & 59 & efe.com & 23 & 15 & 71 \\
\hline 60 & economist.com & 10 & 6 & 19 & 60 & cope.es & 11 & 7 & 70 \\
\hline 61 & elnacional.cat & 10 & 6 & 41 & 61 & ctxt.es & 15 & 9 & 66 \\
\hline 62 & elperiodi.co & 10 & 6 & 157 & 62 & open.spotify.com & 8 & 5 & 65 \\
\hline 63 & as.com & 9 & 6 & 429 & 63 & lainformacion.com & 16 & 10 & 62 \\
\hline 64 & ccma.cat & 9 & 6 & 18 & 64 & malagahoy.es & 5 & 3 & 61 \\
\hline 65 & elnortedecastilla.es & 9 & 6 & 1.328 & 65 & zendalibros.com & 14 & 9 & 60 \\
\hline 66 & larazon.es & 9 & 6 & 82 & 66 & atresplayer.com & 7 & 4 & 55 \\
\hline 67 & lasprovincias.es & 9 & 6 & 80 & 67 & facebook.com & 17 & 11 & 51 \\
\hline 68 & laverdad.es & 9 & 6 & 321 & 68 & cat.elpais.com & 8 & 5 & 50 \\
\hline 69 & letraslibres.com & 9 & 6 & 13 & 69 & ddsevilla.info & 4 & 3 & 50 \\
\hline 70 & theobjective.com & 9 & 6 & 25 & 70 & levante-emv.com & 5 & 3 & 49 \\
\hline 71 & cat.elpais.com & 8 & 5 & 50 & 71 & rtve.es & 23 & 15 & 43 \\
\hline 72 & google.com & 8 & 5 & 15 & 72 & bbc.com & 15 & 9 & 43 \\
\hline 73 & lainfo.news & 8 & 5 & 20 & 73 & elnacional.cat & 10 & 6 & 41 \\
\hline 74 & laopiniondemalaga.es & 8 & 5 & 35 & 74 & ideal.es & 7 & 4 & 39 \\
\hline 75 & lemonde.fr & 8 & 5 & 18 & 75 & elcomercio.es & 4 & 3 & 38 \\
\hline 76 & maldita.es & 8 & 5 & 10 & 76 & elmundotoday.com & 18 & 11 & 37 \\
\hline 77 & Moncloa.com & 8 & 5 & 12 & 77 & naiz.eus & 5 & 3 & 36 \\
\hline 78 & mundodeportivo.com & 8 & 5 & 33 & 78 & ft.com & 17 & 11 & 35 \\
\hline 79 & niemanlab.org & 8 & 5 & 15 & 79 & laopiniondemalaga.es & 8 & 5 & 35 \\
\hline 80 & open.spotify.com & 8 & 5 & 65 & 80 & medium.com & 15 & 9 & 33 \\
\hline 81 & periodistadigital.com & 8 & 5 & 10.505 & 81 & mundodeportivo.com & 8 & 5 & 33 \\
\hline 82 & vilaweb.cat & 8 & 5 & 2.725 & 82 & reuters.com & 12 & 8 & 32 \\
\hline 83 & agendapublica.elperiodico.com & 7 & 4 & 13 & 83 & blogs.grupojoly.com & 7 & 4 & 32 \\
\hline 84 & atresplayer.com & 7 & 4 & 55 & 84 & diariovasco.com & 6 & 4 & 32 \\
\hline 85 & blogs.grupojoly.com & 7 & 4 & 32 & 85 & amp.elmundo.es & 13 & 8 & 31 \\
\hline 86 & diariodecadiz.es & 7 & 4 & 1.127 & 86 & ivoox.com & 7 & 4 & 31 \\
\hline 87 & dircomfidencial.com & 7 & 4 & 16 & 87 & google.es & 18 & 11 & 30 \\
\hline 88 & dozz.es & 7 & 4 & 112 & 88 & europapress.es & 19 & 12 & 29 \\
\hline 89 & ideal.es & 7 & 4 & 39 & 89 & apmadrid.es & 10 & 6 & 27 \\
\hline 90 & ivoox.com & 7 & 4 & 31 & 90 & cnn.it & 14 & 9 & 25 \\
\hline 91 & jotdown.es & 7 & 4 & 10 & 91 & theobjective.com & 9 & 6 & 25 \\
\hline 92 & m.youtube.com & 7 & 4 & 14 & 92 & okdiario.com & 11 & 7 & 22 \\
\hline 93 & time.com & 7 & 4 & 10 & 93 & elecciones.eldiario.es & 5 & 3 & 22 \\
\hline 94 & ver.20m.es & 7 & 4 & 10 & 94 & meneame.net & 4 & 3 & 21 \\
\hline 95 & ww.cope.es & 7 & 4 & 9 & 95 & lainfo.news & 8 & 5 & 20 \\
\hline 96 & abcblogs.abc.es & 6 & 4 & 84 & 96 & nzzl.us & 6 & 4 & 20 \\
\hline 97 & bloom.bg & 6 & 4 & 13 & 97 & poynter.org & 5 & 3 & 20 \\
\hline 98 & change.org & 6 & 4 & 6 & 98 & wsj.com & 4 & 3 & 20 \\
\hline 99 & cjr.org & 6 & 4 & 16 & 99 & economist.com & 10 & 6 & 19 \\
\hline 100 & clar.in & 6 & 4 & 8 & 100 & axios.com & 4 & 3 & 19 \\
\hline 101 & clarin.com & 6 & 4 & 6 & 101 & ccma.cat & 9 & 6 & 18 \\
\hline 102 & diariovasco.com & 6 & 4 & 32 & 102 & lemonde.fr & 8 & 5 & 18 \\
\hline 103 & elsaltodiario.com & 6 & 4 & 7 & 103 & directa.cat & 4 & 3 & 18 \\
\hline 104 & link.medium.com & 6 & 4 & 11 & 104 & scl.io & 4 & 3 & 18 \\
\hline 105 & Irzn.es & 6 & 4 & 2.153 & 105 & Ine.es & 4 & 3 & 17 \\
\hline
\end{tabular}


Tabla 2. Dominios compartidos por periodistas en España (junio 2018 - mayo 2019).

\begin{tabular}{|c|c|c|c|c|c|c|c|c|c|}
\hline \multicolumn{10}{|c|}{158 miembros } \\
\hline \multicolumn{5}{|c|}{ Ordenado por número de miembros que han visitado el dominio } & \multicolumn{5}{|c|}{ Ordenado por número de veces que se ha visitado el dominio } \\
\hline \multicolumn{2}{|c|}{ Dominios } & \multirow{2}{*}{$\frac{\text { Miembros }}{6}$} & \multirow{2}{*}{$\frac{\%}{4}$} & \multirow{2}{*}{$\frac{\text { Veces }}{7}$} & \multicolumn{2}{|r|}{ Dominios } & \multirow{2}{*}{$\frac{\text { Miembros }}{4}$} & \multirow{2}{*}{$\frac{\%}{3}$} & \multirow{2}{*}{$\frac{\text { Veces }}{17}$} \\
\hline 106 & m.facebook.com & & & & 106 & techcrunch.com & & & \\
\hline 107 & nzzl.us & 6 & 4 & 20 & 107 & cincodias.elpais.com & 13 & 8 & 16 \\
\hline 108 & ondace.ro & 6 & 4 & 79 & 108 & dircomfidencial.com & 7 & 4 & 16 \\
\hline 109 & qz.com & 6 & 4 & 12 & 109 & cjr.org & 6 & 4 & 16 \\
\hline 110 & 24matins.es & 5 & 3 & 6 & 110 & libremercado.com & 4 & 3 & 16 \\
\hline 111 & amp.elperiodico.com & 5 & 3 & 15 & 111 & google.com & 8 & 5 & 15 \\
\hline 112 & blogs.20minutos.es & 5 & 3 & 5 & 112 & niemanlab.org & 8 & 5 & 15 \\
\hline 113 & congreso.es & 5 & 3 & 5 & 113 & amp.elperiodico.com & 5 & 3 & 15 \\
\hline 114 & diarioinformacion.com & 5 & 3 & 5 & 114 & elmon.cat & 5 & 3 & 15 \\
\hline 115 & elcomidista.elpais.com & 5 & 3 & 7 & 115 & libertaddigital.com & 4 & 3 & 15 \\
\hline 116 & elecciones.eldiario.es & 5 & 3 & 22 & 116 & lavozdegalicia.es & 12 & 8 & 14 \\
\hline 117 & elmon.cat & 5 & 3 & 15 & 117 & m.youtube.com & 7 & 4 & 14 \\
\hline 118 & elperiodico.cat & 5 & 3 & 5 & 118 & verne.elpais.com & 12 & 8 & 13 \\
\hline 119 & google.co.uk & 5 & 3 & 5 & 119 & letraslibres.com & 9 & 6 & 13 \\
\hline 120 & guerraeterna.com & 5 & 3 & 5 & 120 & agendapublica.elperiodico.com & 7 & 4 & 13 \\
\hline 121 & haaretz.com & 5 & 3 & 11 & 121 & bloom.bg & 6 & 4 & 13 \\
\hline 122 & lavozdigital.es & 5 & 3 & 75 & 122 & Moncloa.com & 8 & 5 & 12 \\
\hline 123 & levante-emv.com & 5 & 3 & 49 & 123 & qz.com & 6 & 4 & 12 \\
\hline 124 & liberation.fr & 5 & 3 & 9 & 124 & eljueves.es & 4 & 3 & 12 \\
\hline 125 & malagahoy.es & 5 & 3 & 61 & 125 & es.rt.com & 4 & 3 & 12 \\
\hline 126 & naiz.eus & 5 & 3 & 36 & 126 & laboratoriodeperiodismo.org & 4 & 3 & 12 \\
\hline 127 & newtral.es & 5 & 3 & 313 & 127 & link.medium.com & 6 & 4 & 11 \\
\hline 128 & play.cadenaser.com & 5 & 3 & 5 & 128 & haaretz.com & 5 & 3 & 11 \\
\hline 129 & poynter.org & 5 & 3 & 20 & 129 & cuartopoder.es & 4 & 3 & 11 \\
\hline 130 & retina.elpais.com & 5 & 3 & 6 & 130 & maldita.es & 8 & 5 & 10 \\
\hline 131 & agendapublica.elpais.com & 4 & 3 & 5 & 131 & jotdown.es & 7 & 4 & 10 \\
\hline 132 & amazon.es & 4 & 3 & 10 & 132 & time.com & 7 & 4 & 10 \\
\hline 133 & axios.com & 4 & 3 & 19 & 133 & ver.20m.es & 7 & 4 & 10 \\
\hline 134 & boe.es & 4 & 3 & 6 & 134 & amazon.es & 4 & 3 & 10 \\
\hline 135 & burgosconecta.es & 4 & 3 & 639 & 135 & esquire.com & 4 & 3 & 10 \\
\hline 136 & catalunyaplural.cat & 4 & 3 & 5 & 136 & granadahoy.com & 4 & 3 & 10 \\
\hline 137 & cuartopoder.es & 4 & 3 & 11 & 137 & variety.com & 4 & 3 & 10 \\
\hline 138 & cuatro.com & 4 & 3 & 6 & 138 & ww.cope.es & 7 & 4 & 9 \\
\hline 139 & ddsevilla.info & 4 & 3 & 50 & 139 & liberation.fr & 5 & 3 & 9 \\
\hline 140 & deia.eus & 4 & 3 & 4 & 140 & elcritic.cat & 4 & 3 & 9 \\
\hline 141 & directa.cat & 4 & 3 & 18 & 141 & m.huffingtonpost.es & 4 & 3 & 9 \\
\hline 142 & dle.rae.es & 4 & 3 & 4 & 142 & clar.in & 6 & 4 & 8 \\
\hline 143 & efectococuyo.com & 4 & 3 & 4 & 143 & fcbarcelona.es & 4 & 3 & 8 \\
\hline 144 & elcatalan.es & 4 & 3 & 4 & 144 & reddit.com & 4 & 3 & 8 \\
\hline 145 & elcomercio.es & 4 & 3 & 38 & 145 & elsaltodiario.com & 6 & 4 & 7 \\
\hline 146 & elcritic.cat & 4 & 3 & 9 & 146 & m.facebook.com & 6 & 4 & 7 \\
\hline 147 & eldiariomontanes.es & 4 & 3 & 302 & 147 & elcomidista.elpais.com & 5 & 3 & 7 \\
\hline 148 & elEconomista.es & 4 & 3 & 4 & 148 & newyorker.com & 4 & 3 & 7 \\
\hline 149 & eljueves.es & 4 & 3 & 12 & 149 & change.org & 6 & 4 & 6 \\
\hline 150 & elpuntavui.cat & 4 & 3 & 111 & 150 & clarin.com & 6 & 4 & 6 \\
\hline 151 & eltemps.cat & 4 & 3 & 4 & 151 & 24matins.es & 5 & 3 & 6 \\
\hline 152 & en.m.wikipedia.org & 4 & 3 & 4 & 152 & retina.elpais.com & 5 & 3 & 6 \\
\hline 153 & es.rt.com & 4 & 3 & 12 & 153 & boe.es & 4 & 3 & 6 \\
\hline 154 & esquire.com & 4 & 3 & 10 & 154 & cuatro.com & 4 & 3 & 6 \\
\hline 155 & farodevigo.es & 4 & 3 & 4 & 155 & lamoncloa.gob.es & 4 & 3 & 6 \\
\hline 156 & fcbarcelona.es & 4 & 3 & 8 & 156 & prnoticias.com & 4 & 3 & 6 \\
\hline 157 & fnpi.org & 4 & 3 & 4 & 157 & vimeo.com & 4 & 3 & 6 \\
\hline 158 & fundeu.es & 4 & 3 & 5 & 158 & blogs.20minutos.es & 5 & 3 & 5 \\
\hline 159 & granadahoy.com & 4 & 3 & 10 & 159 & congreso.es & 5 & 3 & 5 \\
\hline
\end{tabular}


Tabla 2. Dominios compartidos por periodistas en España (junio 2018 - mayo 2019).

\begin{tabular}{|c|c|c|c|c|c|c|c|c|c|}
\hline \multicolumn{10}{|c|}{158 miembros } \\
\hline \multicolumn{5}{|c|}{ Ordenado por número de miembros que han visitado el dominio } & \multicolumn{5}{|c|}{ Ordenado por número de veces que se ha visitado el dominio } \\
\hline \multicolumn{2}{|c|}{ Dominios } & \multirow{2}{*}{$\frac{\text { Miembros }}{4}$} & \multirow{2}{*}{$\frac{\%}{3}$} & \multirow{2}{*}{$\frac{\text { Veces }}{76}$} & \multicolumn{2}{|c|}{ Dominios } & \multirow{2}{*}{$\frac{\text { Miembros }}{5}$} & \multirow{2}{*}{$\frac{\%}{3}$} & \multirow{2}{*}{$\frac{\text { Veces }}{5}$} \\
\hline 160 & hoy.es & & & & 160 & diarioinformacion.com & & & \\
\hline 161 & huffp.st & 4 & 3 & 4 & 161 & elperiodico.cat & 5 & 3 & 5 \\
\hline 162 & imdb.com & 4 & 3 & 5 & 162 & google.co.uk & 5 & 3 & 5 \\
\hline 163 & juntadeandalucia.es & 4 & 3 & 5 & 163 & guerraeterna.com & 5 & 3 & 5 \\
\hline 164 & kiosko.net & 4 & 3 & 4 & 164 & play.cadenaser.com & 5 & 3 & 5 \\
\hline 165 & laboratoriodeperiodismo.org & 4 & 3 & 12 & 165 & agendapublica.elpais.com & 4 & 3 & 5 \\
\hline 166 & lamoncloa.gob.es & 4 & 3 & 6 & 166 & catalunyaplural.cat & 4 & 3 & 5 \\
\hline 167 & libertaddigital.com & 4 & 3 & 15 & 167 & fundeu.es & 4 & 3 & 5 \\
\hline 168 & libremercado.com & 4 & 3 & 16 & 168 & imdb.com & 4 & 3 & 5 \\
\hline 169 & Ine.es & 4 & 3 & 17 & 169 & juntadeandalucia.es & 4 & 3 & 5 \\
\hline 170 & m.europapress.es & 4 & 3 & 4 & 170 & merca2.es & 4 & 3 & 5 \\
\hline 171 & m.huffingtonpost.es & 4 & 3 & 9 & 171 & nobbot.com & 4 & 3 & 5 \\
\hline 172 & meneame.net & 4 & 3 & 21 & 172 & deia.eus & 4 & 3 & 4 \\
\hline 173 & merca2.es & 4 & 3 & 5 & 173 & dle.rae.es & 4 & 3 & 4 \\
\hline 174 & newyorker.com & 4 & 3 & 7 & 174 & efectococuyo.com & 4 & 3 & 4 \\
\hline 175 & nobbot.com & 4 & 3 & 5 & 175 & elcatalan.es & 4 & 3 & 4 \\
\hline 176 & nymag.com & 4 & 3 & 4 & 176 & elEconomista.es & 4 & 3 & 4 \\
\hline 177 & premiosnh.com & 4 & 3 & 4 & 177 & eltemps.cat & 4 & 3 & 4 \\
\hline 178 & prnoticias.com & 4 & 3 & 6 & 178 & en.m.wikipedia.org & 4 & 3 & 4 \\
\hline 179 & redaccionmedica.com & 4 & 3 & 4 & 179 & farodevigo.es & 4 & 3 & 4 \\
\hline 180 & reddit.com & 4 & 3 & 8 & 180 & fnpi.org & 4 & 3 & 4 \\
\hline 181 & revista5w.com & 4 & 3 & 4 & 181 & huffp.st & 4 & 3 & 4 \\
\hline 182 & scl.io & 4 & 3 & 18 & 182 & kiosko.net & 4 & 3 & 4 \\
\hline 183 & servimedia.es & 4 & 3 & 241 & 183 & m.europapress.es & 4 & 3 & 4 \\
\hline 184 & techcrunch.com & 4 & 3 & 17 & 184 & nymag.com & 4 & 3 & 4 \\
\hline 185 & vanitatis.elconfidencial.com & 4 & 3 & 4 & 185 & premiosnh.com & 4 & 3 & 4 \\
\hline 186 & variety.com & 4 & 3 & 10 & 186 & redaccionmedica.com & 4 & 3 & 4 \\
\hline 187 & vimeo.com & 4 & 3 & 6 & 187 & revista5w.com & 4 & 3 & 4 \\
\hline 188 & wsj.com & 4 & 3 & 20 & 188 & vanitatis.elconfidencial.com & 4 & 3 & 4 \\
\hline Tota & & & & & & & & & 60.227 \\
\hline
\end{tabular}

Fuente: Elaboración propia (2020)

Youtube e Instagram son las redes más compartidas en el grupo de los Periodistas. Youtube en segunda posición e Instagram en la octava. Facebook, sin embargo, no aparece hasta la posición 30a y está por detrás del buscador google (posición 28a). En la posición 34a aparece Linkedin.

Prácticamente todos los dominios compartidos por los periodistas pertenecen a medios de comunicación o blogs vinculados a medios o empresas periodísticas o de comunicación. Los que no lo son constituyen una proporción inapreciable (menos del 1\%) y son compartidos por muy pocos miembros. Los dominios a las instituciones también tienen muy poca presencia en los compartidos por los periodistas: lamoncloa.gob.es ocupa la posición 166a, Congreso.es, la 113a y boe.es, la 134a. Es destacable que los periodistas no han compartido ni un solo dominio de partidos o grupos del Congreso español.

Los periodistas han compartido 188 dominios un total de 60.227 veces. Las veces que esta compartición no ha sido a un medio o a un ente relacionado con la comunicación o el periodismo o a una red social como Youtube, Instagram, Facebook o Linkedin suman solo 377, una cifra inapreciable (0,6\%).

En cuanto a la presencia de dominios de medios extranjeros, aparecen de manera destacada nytimes.com en posición 14a, compartido por 34 miembros, y theguardian.com en la 17a, compartido por 
30 miembros. Más abajo en el ranking aparecen bastantes webs de medios no españoles: The Washington Post (posición 29a), Financial Times (31a), BBC (35a), CNN (40a), Reuters (50a), The Economist (60a), Le Monde (75a), y más alejados, Time, Bloomberg, Russia Today, Variety, Clarín, Haaretz, Libération, Wall Street Journal, The New Yorker y New York Magazine.

\section{Comparación entre diputados y periodistas}

La coincidencia entre los dominios que comparten los diputados y los periodistas es notable. En ambos casos los más presentes y en primeras posiciones son los periódicos convencionales españoles y los medios nativos digitales más difundidos. Las diferencias son inapreciables. En cuanto al uso de redes sociales, los periodistas utilizan Instagram mucho más que los diputados y, en cambio, no utilizan Facebook tanto como ellos (Gráficos 1 y 2).

Los diputados no comparten prácticamente enlaces a medios no españoles. La presencia de enlaces a medios extranjeros es mucho menor entre los diputados que entre los periodistas, tanto por el número de medios compartidos como por el número de miembros que los comparten y las veces que lo hacen.

Los enlaces a las webs de los grupos políticos o a instituciones son prácticamente inexistentes entre los periodistas, mientras que sí aparecen, pero no de manera destacable, entre los diputados. Sin embargo, se debe reseñar que en este último grupo la compartición de dominios vinculados al PP destaca por encima del resto de grupos.

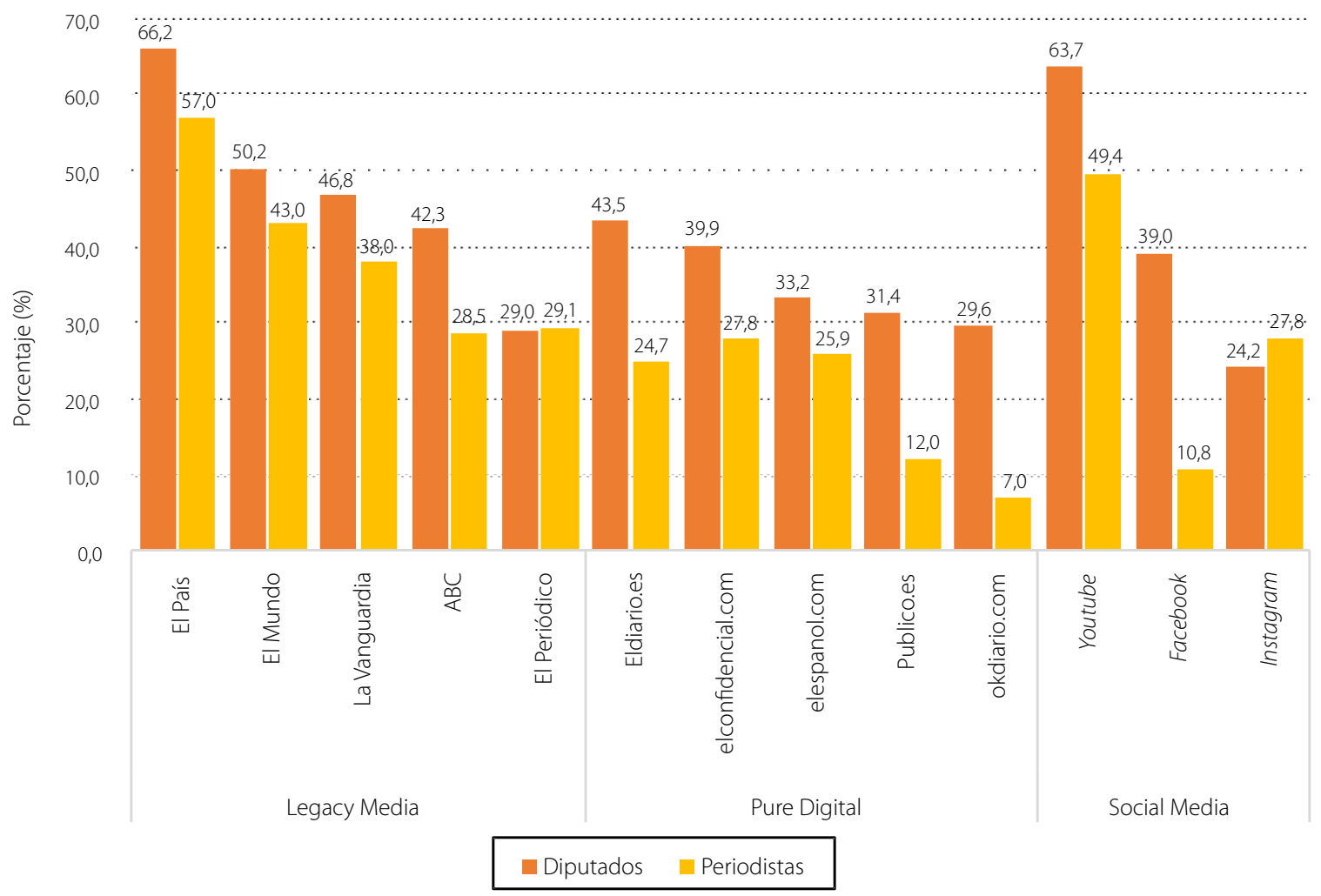

Gráfico 1. Dominios compartidos por diputados y periodistas. Perfiles (junio 2018 - mayo 2019).

Fuente: Elaboración propia (2020). 



\section{Conclusión}

La mayor parte de los dominios compartidos por los miembros del Congreso corresponden a medios de comunicación. Es escaso el uso de enlaces a partidos, instituciones del Estado o páginas web de otras entidades u organismos. Por tanto, se establece un grado de dependencia de los medios por parte de los políticos para difundir su mensaje: no proporcionan en su interacción con los usuarios enlaces a fuentes o recursos propios (blog personal, página web propia... ), sino que casi exclusivamente reproducen el mensaje que procede de los medios. A la vez, los directores de medios y los periodistas reemiten los mensajes que proceden de los políticos, con lo que se produce una relación endogámica, un bucle autorreferencial formado por políticos y periodistas que se retroalimenta a sí mismo y limita la presencia de mensajes que rompan con esta dinámica.

Esta relación endogámica se da mucho más en el colectivo de periodistas, ya que si bien los diputados comparten muy poco los dominios de sus propios partidos (a excepción del PP), los periodistas sí difunden sus propios productos o los de sus colegas de profesión.

Se confirma, por tanto, una conclusión ya obtenida en estudios precedentes referenciados en este artículo: parecería que las redes sociales no fomentan el diálogo, la interacción ni la apertura al hipertexto, sino que en el caso de los políticos (diputados) y periodistas, la actividad que llevan a cabo en Twitter a la hora de compartir enlaces adopta la forma de un bucle autorreferencial que se alimenta a sí mismo y repite el mismo mensaje de las mismas fuentes a los mismos destinatarios. Estamos ante una nueva muestra del limitado uso de las oportunidades del Web 2.0 y las redes sociales.

Esta endogamia se da también de manera geográica y lingüística. Los diputados no comparten enlaces a medios no españoles: la presencia de medios extranjeros es prácticamente inapreciable, un 6\%. En el caso de los periodistas, esta presencia es un poco mayor (15\%), pero si observamos las veces que se comparten enlaces a medios no españoles, la cifra es casi insignificante si dejamos de lado medios como el New York Times, The Guardian o el Washington Post.

La red social más compartida es Facebook, seguida de Youtube. En el periodo estudiado, los miembros del Congreso comparten el dominio de Instagram, pero en mucha menor medida que las otras dos redes sociales y haciendo de ella un uso mucho menor que la población española. En cambio, los periodistas utilizan mucho más Instagram y mucho menos Facebook que los diputados, en lo que es una tendencia más cercana a la sociedad que los rodea.

De los datos presentados en este estudio se obtiene una última conclusión: los medios nativos digitales tienen una presencia relevante entre los referentes de los políticos, pero los convencionales siguen teniendo todavía un gran peso, principalmente los periódicos impresos. Los principales diarios españoles como El País, El Mundo, La Vanguardia o ABC siguen siendo los referentes de los políticos y los que estos perciben como principales formadores de opinión, cuando en realidad existe un universo digital muy alejado de estos grandes medios, los cuales han dejado de ser referentes para la mayor parte de los usuarios de las redes sociales.

\section{Colaboradores}

Los dos autores han contribuido por igual en el proceso de planteamiento del artículo, elaboración del estado del arte, formulación de hipótesis, confección del trabajo de campo, discusión de resultados, redacción del texto y conclusiones del estudio.

\section{Referencias}

Adams, A.; McCorkindale, T. Dialogue and transparency: a content analysis of how the 2012 presidential candidates use
Twitter. Public Relations Review, v. 39, n. 4 p. 357-359, 2013. Doi: https://doi.org/10.1016/j.pubrev.2013.07.016. 
Aharony, N. Twitter use by three political leaders: an exploratory analysis. Online Information Review, v. 36, n. 4 p. 587-603, 2012. Doi: https://doi.org/10.1108/14684521211254086.

Asociación para la Investigación de Medios de Comunicación. Resumen general de resultados EGM. Madrid: AIMC, 2019. Disponible en: http://reporting.aimc.es/index.htm/\#/main/ cockpit. Acceso en: 1 abr. 2020.

Alonso-Muñoz, L.; Casero-Ripollés, A. Comunicación de los líderes populistas europeos en Twitter: construcción de la agenda y efecto "más es menos". El Profesional de la Información, v. 27, n. 6, p. 1193-1202, 2018. Doi: https://doi. org/10.3145/epi.2018.nov.03.

Ausserhofer, J.; Maireder, A. National Politics on Twitter. Information, Communication and Society, v. 16, n. 3, p. 291-314, 2013. Doi: http://dx.doi.org/10.1080/1369118X.2012.756050.

Bell, E. Facebook is eating the world. Columbia Journalism Review, New York, Mar. 7, 2016. Available from: https://www. cjr.org/analysis/facebook_and_media.php. Cited: Apr. 9, 2020.

Bentivegna, S.; Marchetti, R. Journalists at a crossroads: are traditional norms and practices challenged by Twitter? Journalism, v. 19, n. 2, p. 270-290, 2018. Doi: https://doi.org/10. $1177 / 1464884917716594$.

Berrocal, S. et al. Media prosumers in Political Communication: politainment on YouTube. Comunicar, v. 22, n. 43, p. 65-72, 2014. Doi: http://dx.doi.org/10.3916/C43-2014-06.

Boyd, M. S. (New) Participatory Framework on YouTube? commenter interaction in US political speeches. Journal of Pragmatics, v. 72, p. 46-58, 2014. Doi: https://doi.org/10.1016/j. pragma.2014.03.002.

Bronstein, J. Like me! Analyzing the 2012 presidential candidates' Facebook pages. Online Information Review, v. 37, n. 2, p. 173-192, 2013. Doi: http://dx.doi.org/10.1108/OIR-012013-0002.

Bruns, A.; Highfield, T. Political networks on Twitter: tweeting the Queensland state election. Information, Communication and Society, v. 16, n. 5, p. 667-691, 2013. Doi: http://dx.doi. org/10.1080/1369118X.2013.782328.

Campos-Domínguez, E. Twitter y la comunicación política. El profesional de la información, v. 26, n. 5 p. 785-793, 2017. Doi: https://doi.org/10.3145/epi.2017.sep.01.

Chadwick, A. The hybrid media system: politics and power. Oxford: Oxford University Press, 2013.

Casero-Ripollés, A.; Sintes-Olivella, M.; Franch, P. The populist political communication style in action: podemos's issues and functions on Twitter during the 2016 Spanish general election. American Behavioral Scientist, v. 61, n. 9, p. 986-1001, 2017. Doi: https://doi.org/10.1177/0002764217707624.

Enli, G.; Simonsen, C.-A. 'Social media logic' meets professional norms: Twitter hashtags usage by journalists and politicians. Information, Communication and Society, v. 21, n. 8, p. 1081-1096, 2018. Doi: https://doi.org/10.1080/1369118X.2017.1301515.

Enli, G.; Skogerbø, E. Personalized campaigns in partycentred politics: Twitter and Facebook as arenas for political communication. Information, Communication and Society, v. 16, n. 5, p. 757-774, 2013. Doi: https://doi. org/10.1080/1369118X.2013.782330.
EPdata. Usuarios de redes sociales en España. Madrid: EPdata, 2020. Disponible en: https://www.epdata.es/datos/usuariosredes-sociales-espana-estudio-iab/382. Acceso en: 20 agosto 2020.

Ernst, N. et al. Extreme parties and populism: an analysis of Facebook and Twitter across six countries. Information, Communication and Society, v. 20, n. 9, p. 1 347-1364, 2017. Doi: https://doi.org/10.1080/1369118X.2017.1329333.

Gainous, J.; Wagner, K. M. Tweeting to power: the social media revolution in American politics. Oxford: Oxford University Press, 2014.

García-Perdomo, V. Colombian journalists on Twitter: opinions, gatekeeping, and transparency in political coverage. International Journal of Communication, v. 11, p. 1574-2596, 2017.

Golbeck, J. et al. Twitter use by the US Congress. Journal of the American Society for Information Science and Technology, v. 61, n. 8, p. 1612-1621, 2010.

Gottfried, J. et al. Trump, Clinton Voters, Divided in Their Main Source of Election News. Pew Research Center, Washington, Jan. 17, 2017. Available from: http://www.journalism. org/2017/01/18/trump-clinton-voters-divided-in-their-mainsource-for-election-news/. Cited: April 7, 2020.

Graham, T. et al. New platform, old habits? Candidates' use of Twitter during the 2010 British and Dutch general election campaigns. New Media and Society, v. 18, n. 5, p. 765-783, 2016. Doi: https://doi.org/10.1177/1461444814546728.

Halpern, D.; Gibbs, J. Social media as a catalyst for online deliberation? Exploring the Affordances of Facebook and YouTube for political expression. Computers in Human Behavior, v. 29, n. 3, p. 1159-1168, 2013. Doi: https://doi.org/10. 1016/j.chb.2012.10.008.

Jackson, N. A.; Lilleker, D. G. Microblogging, constituency service and impression management: UK MPs and the use of Twitter. Journal of Legislative Studies, v. 17, n. 1, p. 86-105, 2011. Doi: https://doi.org/10.1080/13572334.2011.545181.

Jungherr, A. Twitter use in election campaigns: a systematic literature review. Journal of Information Technology and Politics, v. 13, n. 1, p. 72-91, 2016. Doi: https://doi.org/10.1080/ 19331681.2015 .1132401$.

Karlsen, R.; Enjolras, B. Styles of social media campaigning and influence in a hybrid political communication system: linking candidate survey data with Twitter data. International Journal of Press/Politics, v. 21, n. 3, p. 338-357, 2016. Doi: https://doi. org/10.1177/1940161216645335.

Lalancette, M.; Raynauld, V. The power of political image: Justin Trudeau, Instagram, and celebrity politics. American Behavioral Scientist, v. 63, n. 7, p. 888-924, 2019. Doi: http:// dx.doi.org/10.1177/0002764217744838.

Larsson, A. O. Top users and long tails: Twitter and Instagram use during the 2015 Norwegian elections. Social Media + Society, v. 3. n. 2, p. 1-12, 2017. Doi: http://dx.doi.org/10. 1177/2056305117713776.

Larsson, A. O. Online all the time? A quantitative assessment of the permanent campaign on Facebook. New Media and Society, v. 18, n. 2, p. 274-292, 2016. Doi: http://dx.doi. org/10.1177/1461444814538798. 
Larsson, A. O. Pandering, protesting, engaging: Norwegian party leaders on Facebook during the 2013 'Short campaign'. Information, Communication and Society, v. 18, n. 4, p. 459-473, 2015. Doi: https://doi.org/10.1080/1369118X.2014.967269.

Lee, B.; Campbell, V. Looking out or turning in? Organizational ramifications of online political posters on Facebook. The International Journal of Press/Politics, v. 21, n. 3, p. 313-337, 2016. Doi: https://doi.org/10.1177/1940161216645928.

Lilleker, D. G. et al. Towards hypermedia campaigning? Perceptions of new media's importance for campaigning by party strategists in comparative perspective. Information, Communication and Society, v. 18, n. 7, p. 747-765, 2015. Doi: http://dx.doi.org/10.1080/1369118X.2014.993679.

López-García, G. New vs 'old' leaderships: the campaign of Spanish general elections 2015 on Twitter. Communication and Society, v. 29, n. 3, p. 149-168, 2016. Doi: http://dx.doi. org/10.15581/003.29.3.149-168.

López-Meri, A.; Casero-Ripollés, A. El debate de la actualidad periodística española en Twitter: del corporativismo de periodistas y políticos al activismo ciudadano. Observatório, v. 10, n. 3, p. 56-79, 2016. Doi: https://doi.org/10.15847/obsOBS 1032016994

López-Merietal. ¿Quéhacen los políticos en Twitter? Funciones y estrategias comunicativas en la campaña electoral española de 2016. El Profesional de la Información, v. 26, n. 5, p. 795-804, 2017. Doi: https://doi.org/10.3145/epi.2017.sep.02.

Mitchell, A. et al. Facebook, top source for political news among millennials. Pew Research Center, Washington, June 1, 2015. Available from: http://www.journalism.org/2015/06/01/ facebook-top-source-for-political-news-among-millennials/. Cited: Apr. 7, 2020.

Molyneux, L.; Mourao, R.-R. Political journalists' normalization of Twitter: interactioon and new affordances. Journalism Studies, v. 20, n. 2, p. 248-266. 2019. Doi: https://doi. org/10.1080/1461670X.2017.1370978.

OJD Interactiva Medios Digitales. Noviembre 2020: tráfico nacional e internacional. Madrid: OJD, 2019. Disponible en: https://www.ojdinteractiva.es/medios-digitales. Acceso en: 1 abr. 2020.

Parmelee, J. H.; Bichard, S. L. Politics and the Twitter revolution: how tweets influence the relationship between political leaders and the public. Lanham: Lexington Books, 2012.

Parmelee, J. H.; Roman, N. Insta-Politicos: motivations for following political leaders on Instagram. Social
Media + Society, v. 5, n. 2, p. 1-12, 2019. Doi: http://dx.doi. org/10.1177/2056305119837662.

Persily, N. Can democracy survive the Internet? Journal of Democracy, v. 28, n. 2, p. 63-76, 2017.

Pérez-Dasilva, J. et al. ¿Dialogan los líderes políticos españoles en Twitter con los medios de comunicación y periodistas? Communication and Society, v. 31, n. 3, p. 299-316, 2018. Doi: https://doi.org/10.15581/003.31.3.299-317.

Pew Research Center. Election 2016: campaigns as a direct source of news. Pew Research Center, Washington, June 18, 2016. Available from: http://www.journalism.org/2016/07/18/ election-2016-campaigns-as-a-direct-source-of-news/. Cited: Apr. 9, 2020.

Russell-Neuman, W. et al. The dynamics of public attention: agenda-setting theory meets big data. Journal of Communication, v. 64, n. 2, p. 193-214, 2014. Doi: http://dx.doi. org/10.1111/jcom.12088.

Russmann, U.; Svensson, J. Interaction on Instagram? Glimpses from the 2014 Swedish Elections. International Journal of e-Politics, v. 8, n. 1, p. 50-66, 2017. Doi: http://dx.doi.org/10. 4018/IJEP.2017010104.

Selva-Ruiz, D.; Caro-Castaño, L. The use of Instagram as a political communication channel by Spanish deputies: the humanization strategy in the "old" and the "new" politics. El Profesional de la Información, v. 26, n. 5, p. 903-915, 2017. Doi: https://doi.org/10.3145/epi.2017.sep.12.

Sørensen, M. P. Political conversations on Facebook: the participation of politicians and citizens. Media, Culture and Society, v. 38, n. 5, p. 664-685, 2016. Doi: https://doi.org/10. $1177 / 0163443715620924$.

Usher, N. et al. Twitter makes it worst: political journalists, gendered echo chambers, and the amplification of gender bias. The International Journal of Press/Politics, v. 23, n. 3, p. 324-344, 2018. Doi: https://doi.org/10.1177/1940161218781254.

van-Kessel, S.; Castelein, R. Shifting the blame: populist politicians' use of Twitter as a tool of opposition. Journal of Contemporary European Research, v. 12, n. 2, p. 594-614, 2016.

Vergeer, M.; Hermans, L. Campaigning on Twitter: microblogging and online social networking as campaign tools in the 2010 general elections in the Netherlands. Journal of Computer-Mediated Communication, v. 18, n. 4, p. 399-419, 2013. Doi: http://dx.doi.org/10.1111/jcc4.12023.

Vergeer, M. et al. Online social networks and micro-blogging in political campaigning: the exploration of a new campaign tool and a new campaign style. Party Politics, v. 19, n. 3, p. 477-501, 2013. Doi: http://dx.doi.org/10.1177/1354068811407580. 